\title{
Revisited Model for Supercooled Large Droplet Impact onto a Solid Surface
}

\author{
P. Trontin* and P. Villedieu* \\ ONERA - The French Aerospace Lab, F-31055 Toulouse, France
}

\begin{abstract}
A rationally based methodology is proposed to derive a new mass loss empirical model for supercooled large droplets. The numerical results from the ONERA two-dimensional trajectory solver and the experimental results from the NASA Papadakis supercooled large droplet database are combined to get both the impinging and the deposited mass flow rates at each point of the test model. These data are used to derive a new model for the collection efficiency. It allows clearly separating the influence of the kinetic energy, which is the dominating effect close to the leading edge, from the influence of the angle of incidence, which is the most influent parameter close to the impingement limits. Moreover, the model can be used for both supercooled large droplets and small droplets.
\end{abstract}

\begin{tabular}{lll} 
& \multicolumn{1}{c}{ Nomenclature } \\
$C a$ & $=$ & capillary number \\
$c$ & $=$ & profile chord, $\mathrm{m}$ \\
$D$ & $=$ & impinging droplet mean mass diameter, $\mathrm{m}$ \\
$K_{n}$ & $=$ & Cossali number based on impinging droplet velocity \\
$K_{0}$ & $=$ & threshold value for $K$ between full deposition and \\
& & splash regimes \\
$\mathrm{LWC}_{\infty}$ & $=$ & freestream liquid water content, $\mathrm{kg} \cdot \mathrm{m}^{-3}$ \\
$\dot{m}_{\mathrm{dep}}$ & $=$ & deposited mass flow rate, $\mathrm{kg} \cdot \mathrm{m}^{-2} \cdot \mathrm{s}^{-1}$ \\
$\dot{m}_{\text {imp }}$ & $=$ & impinging mass flow rate $\mathrm{kg} \cdot \mathrm{m}^{-2} \cdot \mathrm{s}^{-1}$ \\
$O h$ & $=$ & Ohnesorge number \\
$s$ & $=$ & curvilinear abscissa $(\mathrm{s}<\mathrm{for} \mathrm{the} \mathrm{pressure} \mathrm{side,} \mathrm{and}$ \\
& & $s>0$ for the suction side $), \mathrm{m}$ \\
$V$ & $=$ & impinging droplet mean mass velocity, $\mathrm{m} \cdot \mathrm{s}{ }^{-1}$ \\
$V_{\infty}$ & $=$ & freestream droplet velocity, $\mathrm{m} \cdot \mathrm{s}^{-1}$ \\
$W e_{n}$ & $=$ & Weber number based on impinging droplet normal \\
$\alpha$ & $=$ & implocity \\
$\beta_{\mathrm{dep}}$ & $=$ & collection efficiency \\
$\beta_{\text {imp }}$ & $=$ & impingement efficiency \\
$\Delta t$ & $=$ & accretion time, $\mathrm{s}$ \\
$\epsilon$ & $=$ & sticking efficiency \\
$\theta$ & $=$ & angle of incidence, deg \\
$\theta_{c}$ & $=$ & critical angle of incidence, deg \\
$\phi$ & $=$ & mass loss coefficient
\end{tabular}

\section{Introduction}

I CING occurs when an aircraft flies through a cloud in which supercooled droplets are suspended with an ambient air temperature below the freezing point. The droplets impinge on the aircraft surfaces and freeze, leading to ice accretion. The resulting change in the aircraft geometry can modify aerodynamic characteristics (loss of lift, rise of drag), can affect the ability of the probes to provide accurate measurements, can clog air intake, or even damage the engine by ice ingestion; in the worst scenario, it can cause a complete loss of the control of the aircraft. The importance of proper ice control for aircraft operation in cold climates was recently highlighted by several in-flight icing events and crashes. In 1994, an

*Research Engineer, Aerodynamics and Energetics Modeling Department, 2, Avenue Edouard Belin.
ATR-72 crashed in Roselawn, Indiana. It was speculated that this accident was due to supercooled large droplets (SLDs) which can impinge beyond the limits of the protection system due to their high inertia. This crash led to a modification of the regulation rules with the definition of the appendix $\mathrm{O}$ [1], which included freezing drizzle [median volume diameter (MVD) $<500 \mu \mathrm{m}$ ] and freezing rain (MVD $>500 \mu \mathrm{m}$ ) icing conditions. To account for these new rules, both experimental and numerical means of compliance need to be modified and validated. To address this problem, several research projects were launched in North America and in Europe during the last decade. Thanks to these works, icing wind tunnels were updated to reproduce SLD conditions and a first set of models was developed: NASA-LEWICE models [2,3]; models for splashing from Honsek and Habashi [4], Honsek et al. [5], and Hospers and Hoeijmakers [6]; and models from ONERA-The French Aerospace Lab (hereafter referred to as ONERA) [7-10] and from Wang et al. [11]. Experimental data (Papadakis et al. [12-15], EXTreme ICing Environment (EXTICE) European project [16]) were produced for both calibrating and validating these new models.

As far as numerical means of compliance are concerned, the modeling of SLDs is very challenging because it requires taking into account several new physical phenomena that do not need to be considered for small droplets (appendix C [1] conditions). The most important one is related to the so-called "mass loss effect." It corresponds to the fact that large droplet impingement is often accompanied by splashing with secondary droplet reemission, and thus a reduction of the amount of water that would have been deposited otherwise. Droplet deformation and droplet breakup due to the effect of aerodynamic forces may also have an influence on droplet trajectories, and thus on the amount of deposited water. However, except for freezing drizzles, the influence of these phenomena seems to be negligible in most cases, as shown in $[8,17]$.

Droplet splashing is involved in several industrial applications (spray cooling, spray coating, automotive and turbojet combustion chambers, etc.) and has received great attention in the research community during the last 30 years $[12,14,18-20]$. Icing applications are specific because they involve aerodynamic flow around the target with very high impact velocities (around $100 \mathrm{~m} / \mathrm{s}$ ) and the whole range of impact angles for the droplets (from 0 deg at the leading edge to $90 \mathrm{deg}$ near the impingement limit). For this reason, dedicated experiments were performed by Papadakis et al. in the NASA Icing Research Tunnel to get a database in representative conditions [12-15]. These experimental data combined with some ideas issued from existing impingement models (Bai and Gosman [18] for impacts at low velocity, Trujillo et al. [21], Mundo et al. [19,22], Yarin and Weiss [20]) were used by several authors $[3,4,6,8,16]$ in order to derive SLD mass loss models for icing applications. Even if these models yield significantly improved collection efficiency predictions when applied to the experiments performed by Papadakis et al. [12-15], they suffer from several important weaknesses: 
Table 1 Test conditions used for the model calibration and assessment

\begin{tabular}{lcccccc}
\hline Type of airfoil & AOA, deg & MVD, $\mu \mathrm{m}$ & $\mathrm{LWC}_{\infty}, \mathrm{g} \cdot \mathrm{m}^{-3}$ & Chord, $\mathrm{m}$ & Velocity, $\mathrm{m} \cdot \mathrm{s}^{-1}$ & Usage \\
\hline MS(1)-317 & 0.0 & $21,79,92,137,168$ & $0.521,0.496,0.22,0.68,0.75$ & 0.9144 & 78.25 & Model calibration \\
NACA23012 & 2.5 & $20,52,111,154,236$ & $0.521,0.2,0.73,0.2,1.89$ & 0.9144 & 78.25 & Model assessment \\
MS(1)-317 & 8.0 & $11,21,92$ & $0.188,0.521,0.22$ & 0.9144 & 78.25 & Model assessment \\
GLC-305 & 1.5 & $79,92,137,168$ & $0.496,0.22,0.68,0.75$ & 0.9144 & 78.25 & Model assessment \\
\hline
\end{tabular}

1) They are purely empirical models without any theoretical background, except the fact that they rely on some relevant dimensionless numbers introduced in former studies on droplet impingement $[20,22]$.

2) In all the papers $[3,4,8,16]$ except [11], there was no explanation on how the proposed mass loss model could be explicitly derived from the experimental data from Papadakis et al. [12-15]. It was only shown that the model allowed improvement of the collection efficiency prediction.

3) There exist large discrepancies among the existing model predictions despite the fact that they are all based on the same experimental database.

In the present paper, using again the experimental database from Papadakis et al. [12-15], our objective is to propose a rationally based methodology to derive a more reliable mass loss model than the existing ones. In particular, this model allows us to clearly separate the influence of the normal kinetic energy, which is the dominating effect close to the leading edge, from the influence of the angle of incidence, which is the most influent parameter close to the impingement limits. Moreover, the model can be used for both SLDs and small droplets.

The paper is organized as follows. In Sec. II, we present a new analysis of the experimental results from Papadakis et al. [12-15]. Section III is devoted to the derivation and the calibration of the new mass loss model; lastly, Sec. IV presents some validation test cases for both collection efficiency and ice shape predictions.

\section{Analysis of the Experimental Results}

\section{A. Short Presentation of the Experimental Database}

The experimental database used for this comparison was taken from several tests performed in the NASA Icing Research Tunnel [12-15]. The tests were performed using a water spray containing a known concentration of blue dye, and the models were covered with a heavyweight blotter paper. The amount of dye was then measured via reflectance spectroscopy using a charge-coupled device camera. Collection efficiencies were determined on different clean and iced airfoils, for several drop size distributions from MVDs of 11 to $236 \mu \mathrm{m}$ and for several angles of attack from 0 to $8 \mathrm{deg}$. The airspeed for all cases was $78 \mathrm{~m} / \mathrm{s}$.

In the present study, we only considered a limited set of 17 test cases among the 117 distinct ones available in the database. Five different running conditions were used for model calibration purposes, and 12 additional ones were used for the model assessment, as detailed in Table 1.

With regard to the droplet size distribution, the 27-bin discretization given in [14] was used for each running condition.
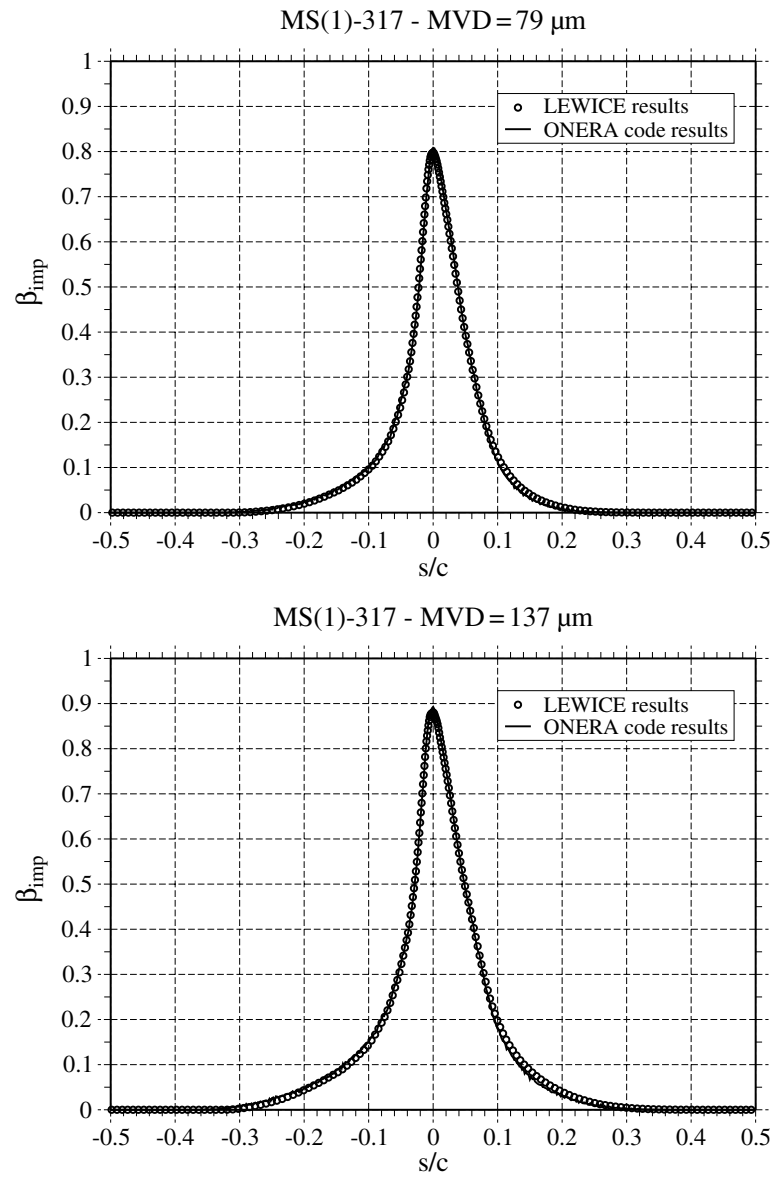

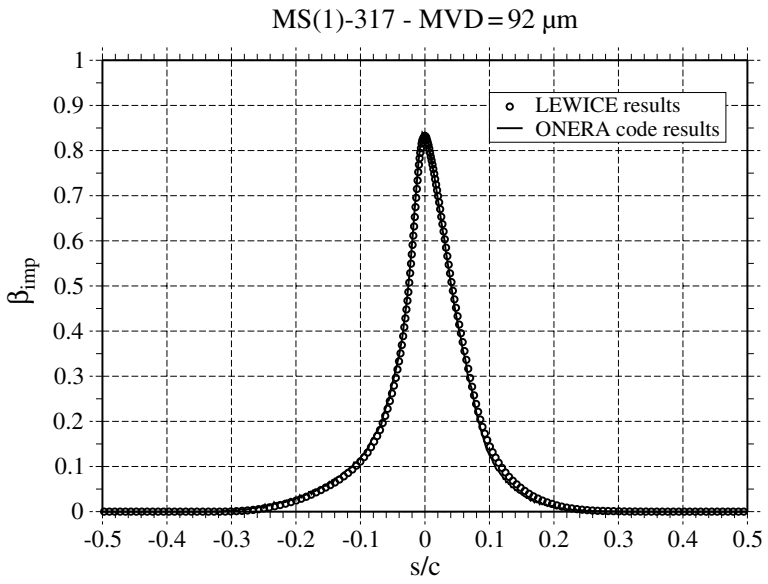

$\operatorname{MS}(1)-317-\operatorname{MVD}=168 \mu \mathrm{m}$

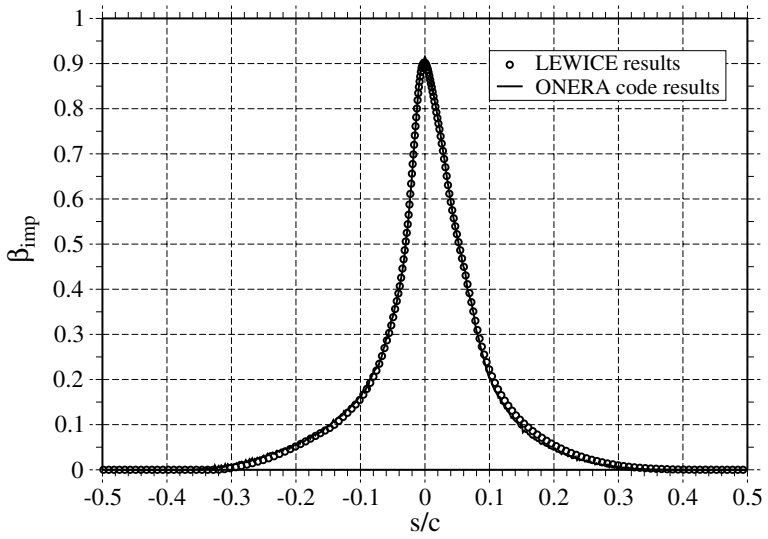

Fig. $1 \beta_{\text {imp }}$ comparison between TRAJ2D (ONERA code) and LEWICE (NASA code). 


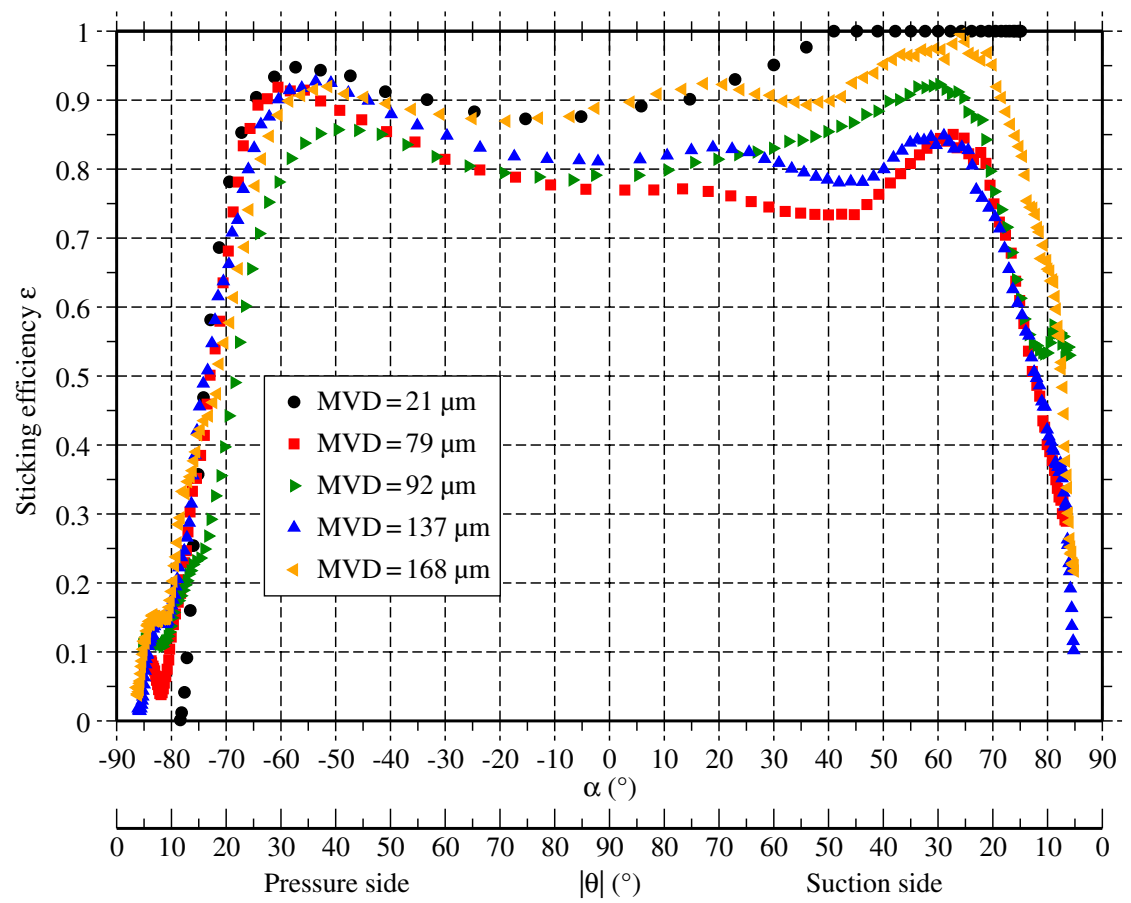

Fig. $2 \epsilon$ against $\alpha$ and $|\theta|$ for the MS(1)-317 profile at $\mathrm{AOA}=0 \mathrm{deg}$.

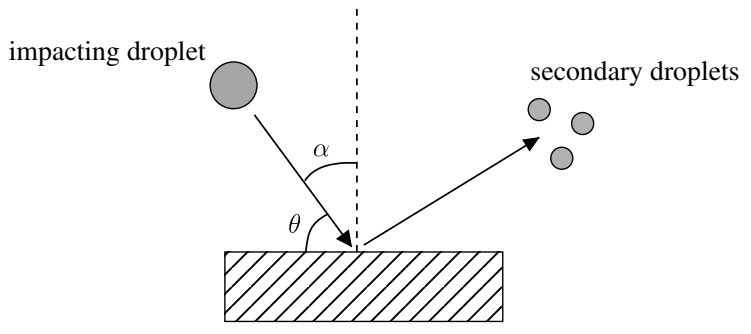

Fig. 3 Definitions of the angle of incidence $\theta$ and the impact angle $\alpha$.

\section{B. Experimental Result Analysis}

The mass loss coefficient $\phi$ is defined as follows:

$$
\phi=\frac{\dot{m}_{\mathrm{imp}}-\dot{m}_{\mathrm{dep}}}{\dot{m}_{\mathrm{imp}}}
$$

where $\dot{m}_{\text {imp }}$ denotes the impinging mass flow rate, and $\dot{m}_{\text {dep }}$ denotes the deposited mass flow rate. Introducing the standard notations for the impingement efficiency and the collection efficiency, respectively,

$\operatorname{MS}(1)-317$

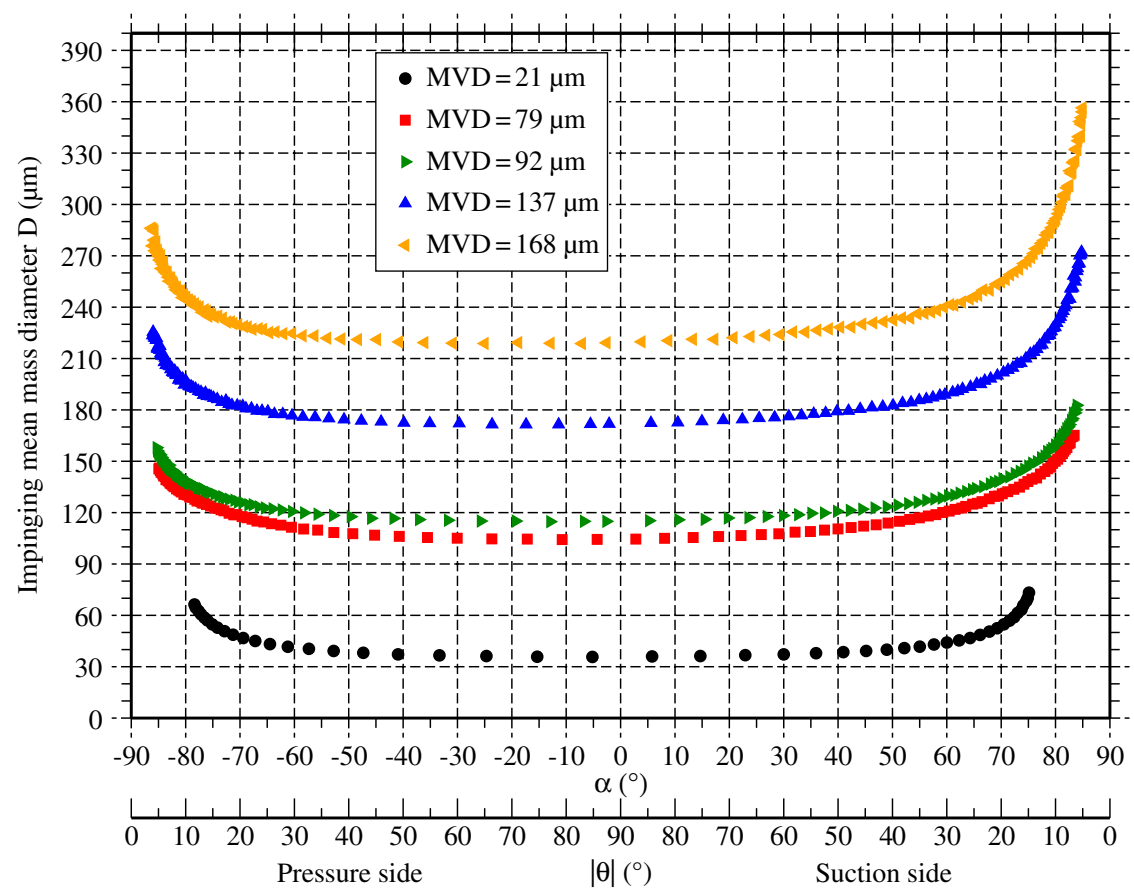

Fig. $4 D$ against $\alpha$ and $|\theta|$ for the $\operatorname{MS}(1)-317$ profile at $\mathrm{AOA}=0 \mathrm{deg}$. 


$$
\beta_{\mathrm{imp}}=\frac{\dot{m}_{\mathrm{imp}}}{\mathrm{LWC}_{\infty} \cdot V_{\infty}}
$$

and

$$
\beta_{\text {dep }}=\frac{\dot{m}_{\text {dep }}}{\mathrm{LWC}_{\infty} \cdot V_{\infty}}
$$

One has

$$
\phi=1-\frac{\beta_{\mathrm{dep}}}{\beta_{\text {imp }}}=1-\epsilon
$$

where, by definition,

$$
\epsilon=\frac{\beta_{\text {dep }}}{\beta_{\text {imp }}}
$$

denotes the sticking efficiency. The quantity that is measured in the experiment, thanks to the blotter paper technique, corresponds to $\beta_{\text {dep. }}$. Because $\beta_{\text {imp }}$ cannot be measured, it is not possible to

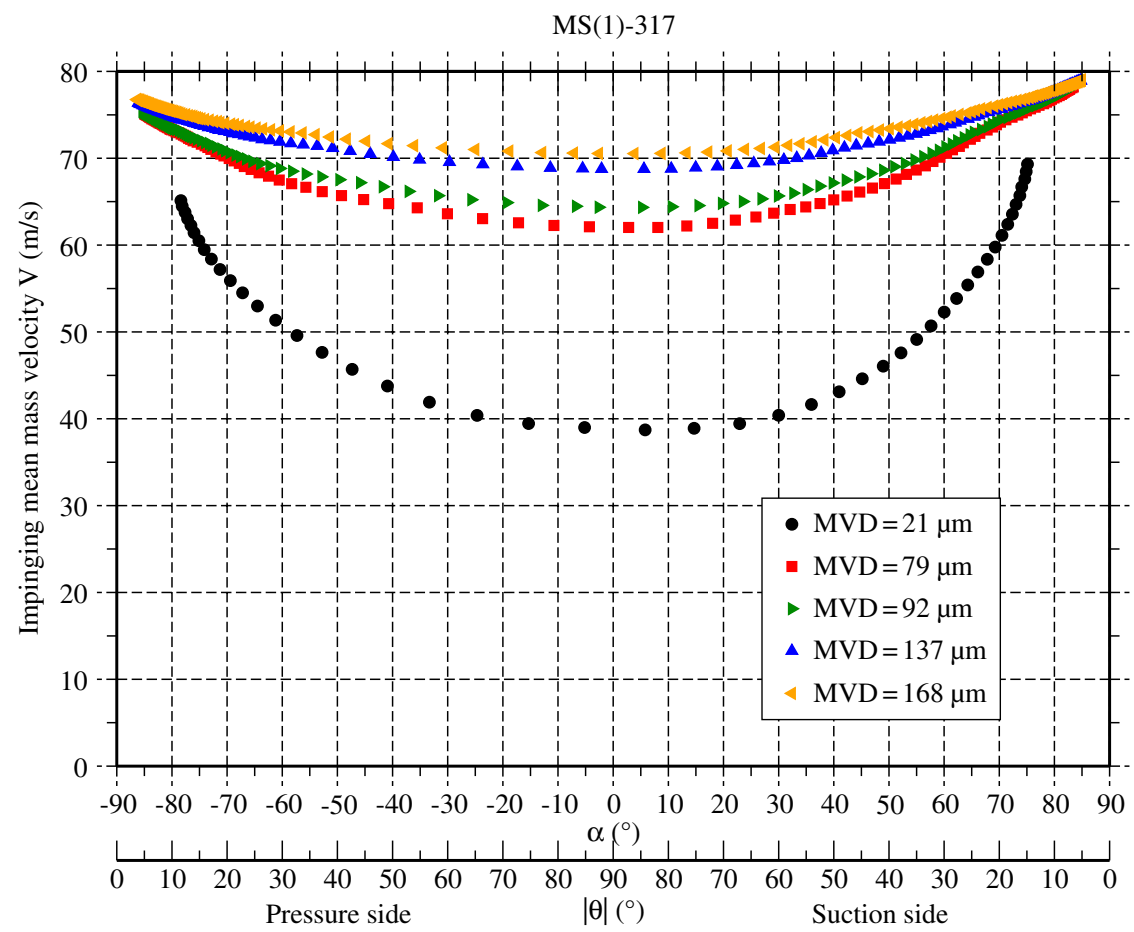

Fig. $5 V$ against $\alpha$ and $|\theta|$ for the MS(1)-317 profile at $\mathrm{AOA}=0 \mathrm{deg}$.

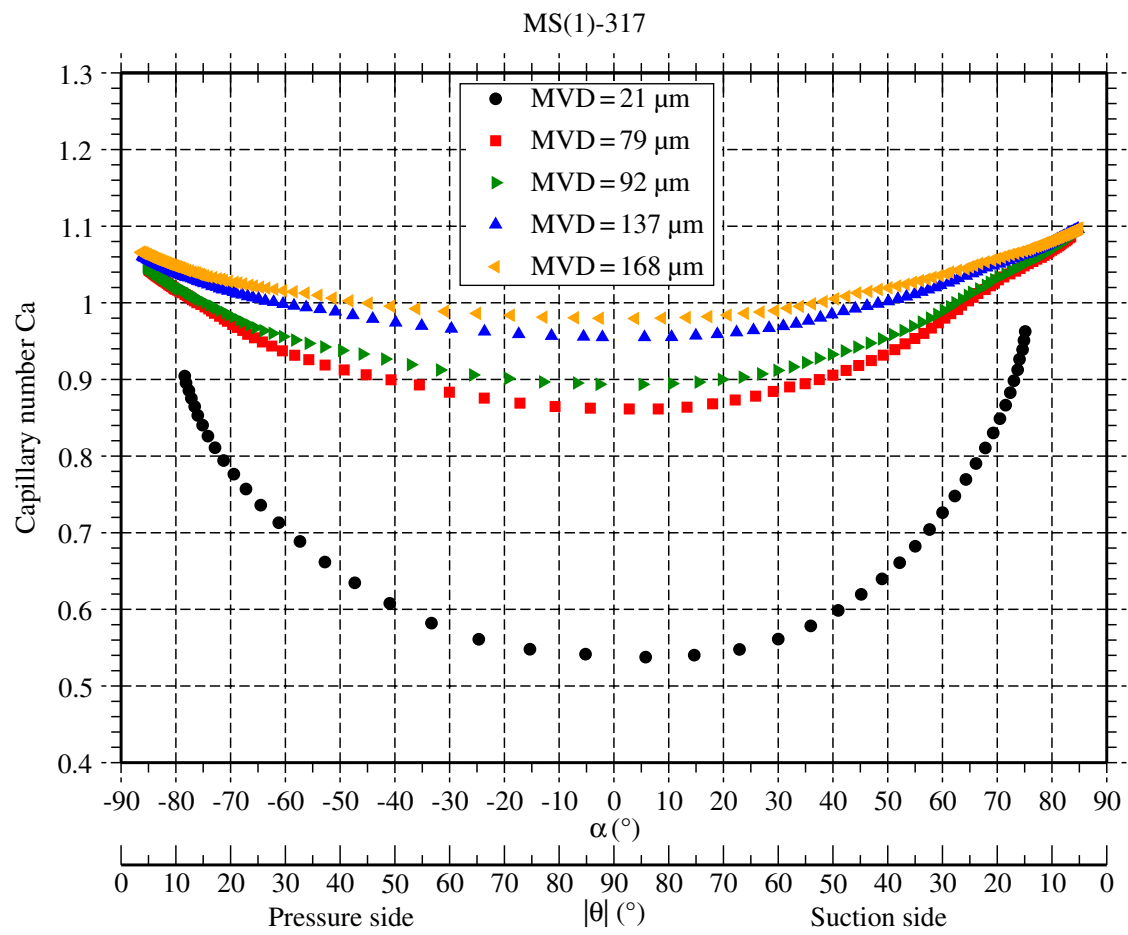

Fig. $6 C a$ against $\alpha$ and $|\theta|$ for the MS(1)-317 profile at $\mathrm{AOA}=0 \mathrm{deg}$. 
Table 2 Model parameters

\begin{tabular}{lc}
\hline Model parameters & Values \\
\hline$K_{0}$ & 657 \\
$\epsilon_{\infty}$ & 0.85 \\
$\tilde{K}_{0}$ & 10 \\
$\theta_{0}$ & $25 \mathrm{deg}$ \\
$\mathrm{Ca} a_{0}$ & 1 \\
$\gamma$ & 1.5 \\
\hline
\end{tabular}

extract the values of the mass loss coefficient from the experimental data. However, $\beta_{\text {imp }}$ can be numerically computed, with quite a good level of accuracy, by using a droplet trajectory solver. Therefore, combining numerical and experimental results, it is possible to simultaneously know the characteristics of the impinging droplets (velocity and diameter), the impinging mass flow rate, the deposited mass flow rate, and the mass loss coefficient at each point of the test model.

We applied this methodology to the clean MS(1)-317 test cases (Table 1). For all the runs, the droplet impingement efficiency $\beta_{\text {imp }}$ was computed using the ONERA two-dimensional (2-D) Lagrangian solver TRAJ2D. The aerodynamic flow solution was obtained with the ONERA 2-D solver EULER2D, where the steady inviscid Euler equations were solved. The influences of droplet deformation and breakup were not taken into account in the trajectory model.
According to previous numerical [8,17] and empirical [23] studies, these effects were significant only for freezing rains $(D>500 \mu \mathrm{m})$. Figure 1 shows a comparison between TRAJ2D and LEWICE results. The agreement is almost perfect (relative error lower than 2\%), which may be considered as a validation of the numerical procedure used in the ONERA solver to compute the impingement efficiency.

Figure 2 shows the variation of the computed sticking efficiency $\epsilon$ with the impact angle $\alpha$ for the MS(1)-317 experiments at an angle of attack (AOA) of 0 deg. The angle of incidence $\theta$ and the impact angle $\alpha$ are defined in Fig. 3. By convention, the positive values of $\alpha$ (or $\theta$ ) correspond to the suction side and the negative values to the pressure side. Even if the results are scattered due to the numerous experimental and numerical uncertainties (droplet cloud homogeneity, droplet size distribution, measurement accuracy near the impingement limit, etc.), two impingement regimes may be clearly distinguished for both the suction and the pressure sides as far as SLDs are concerned (MVD $>50 \mu \mathrm{m}$ ).

1) For small angles of incidence (corresponding to large $\alpha$ ), the sticking efficiency is an increasing function of $|\theta|=90 \mathrm{deg}-|\alpha|$, which tends to zero when $|\theta|$ is close to 0 deg (impingement limit).

2) For intermediate and large angles of incidence, the sticking efficiency does not strongly depend on $\theta$, and even seems to be almost constant in several cases. No clear tendencies can be inferred, except that $\epsilon$ exhibits some kind of "plateau."

It is also worth noticing that the critical angle $\theta_{c}$ separating the two regimes is almost the same (close to $30 \mathrm{deg}$ ) in any SLD cases, whatever the value of the droplet cloud MVD, which varies
$\operatorname{MS}(1)-317$

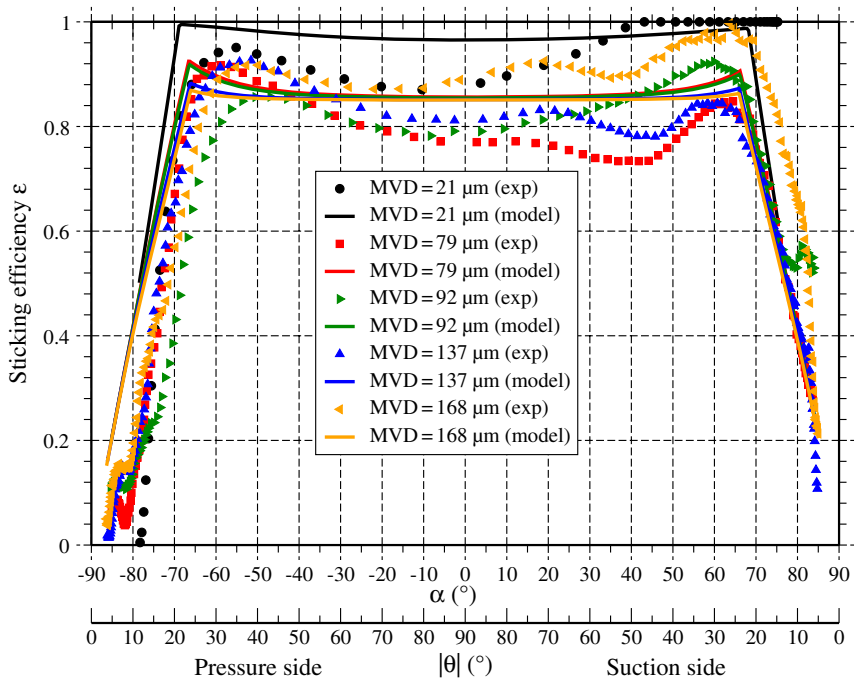

NACA23012

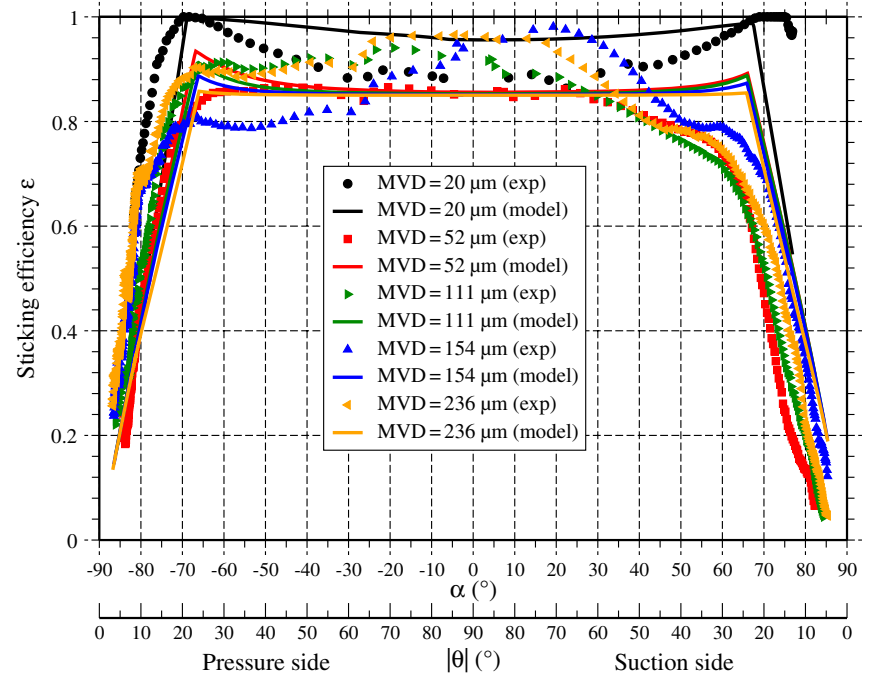

GLC305

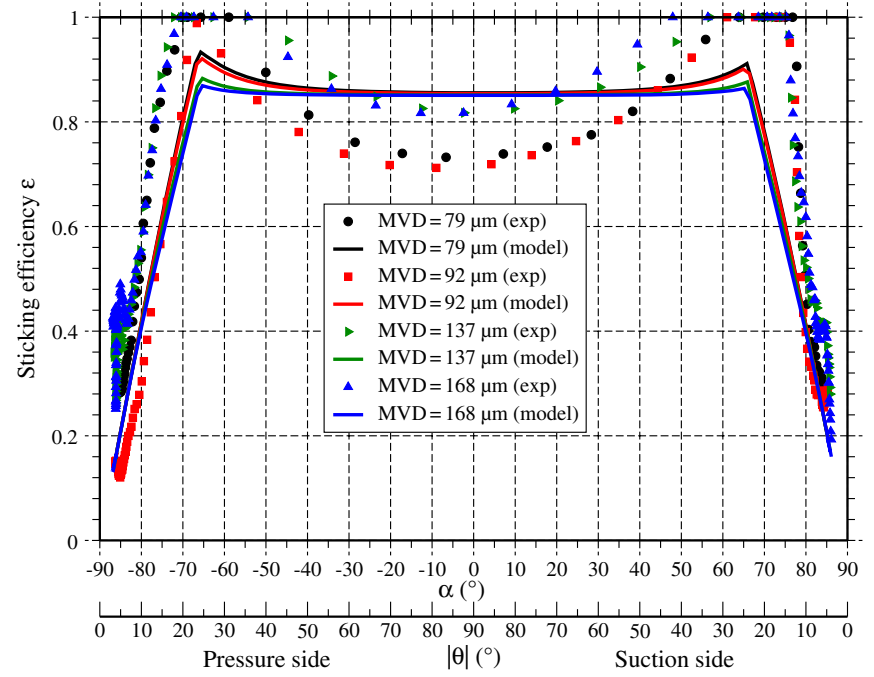

Fig. $7 \epsilon$ against $\alpha$ and $|\theta|$. Comparison between experimental (exp) results (symbols) and the present SLD model (solid lines). 
significantly from 21 to $168 \mu \mathrm{m}$. However, it is worth mentioning that, for the smallest droplet case $(\mathrm{MVD}=21 \mu \mathrm{m})$, the impingement mass rate for the small incidence angles is very low. Because of that, the sticking efficiency estimate is not accurate and may even exceed one, as is the case here for $|\alpha|>40 \mathrm{deg}$.

From a physical point of view, the existence of two different impact regimes may be explained as follows:

1) For small angles of incidence (large $|\alpha|$ ), the capillary forces (which tend to promote droplets sticking to the wall) are balanced by the tangential inertial forces (which tend to tear the droplet out of the wall). Since a part of the droplet is not attached to the wall during the impact process, the tangential kinetic energy is not efficiently dissipated by the viscous forces and the droplet may partially (and even completely) bounce off the wall.
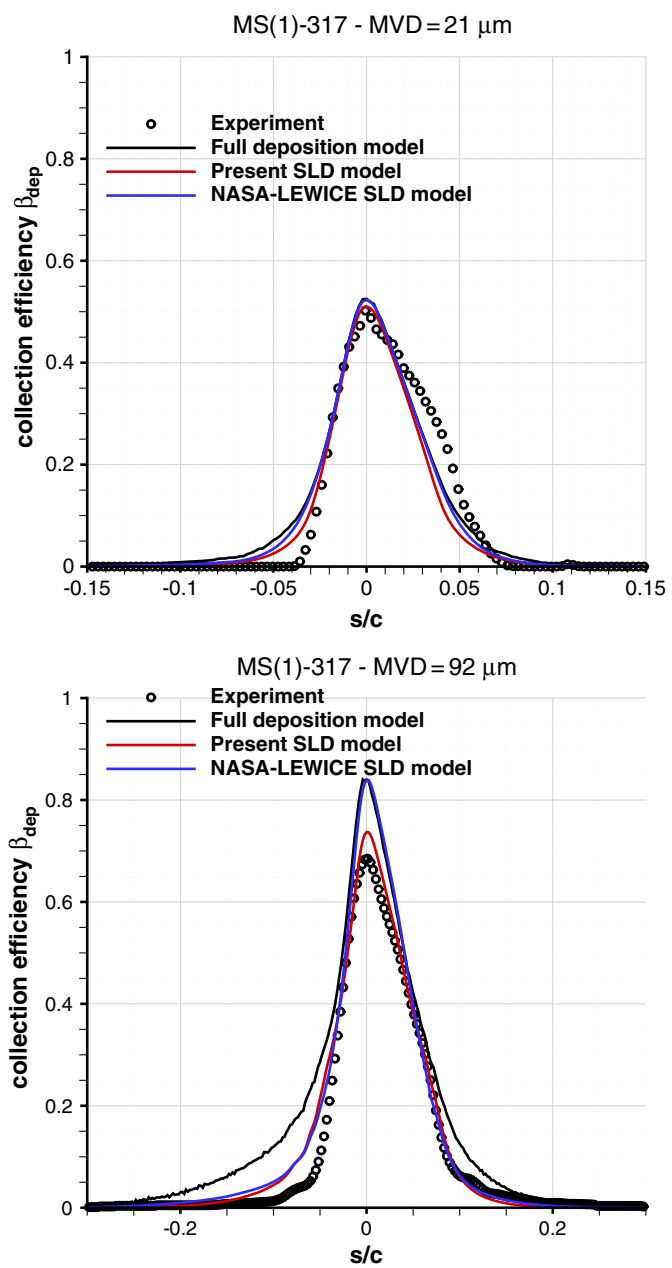

2) When $|\theta|$ increases (which corresponds to a decrease of $|\alpha|$ ), the droplet spreads more onto the wall. This leads to an increase in the dissipation effects due to the viscous forces and in parallel to a decrease in the inertial forces that stretch the droplet and that may break it. Thus, for $|\theta|$ larger than a critical value $\theta_{c}$, the capillary forces are strong enough compared to the inertial forces to maintain the largest part of the droplet deposited on the wall. This could be the origin of the second regime.

As far as the critical angle $\theta_{c}$ is concerned, it seems obvious that it should at least depend on the droplet total velocity and diameter because it is related to the balance among the inertial, capillary, and viscous forces. Figure 4 shows the variation of the impinging droplet mean mass diameter against the impact angle $D(\alpha)$, which is defined as

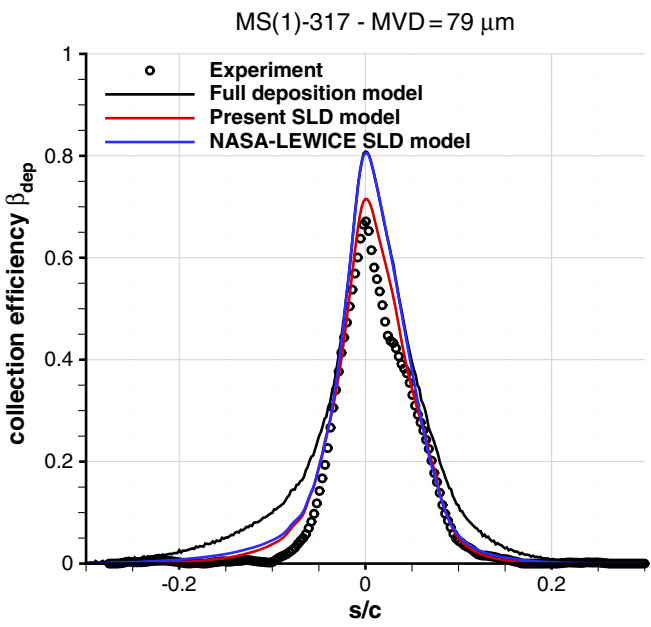

MS(1)-317 - MVD $=137 \mu \mathrm{m}$

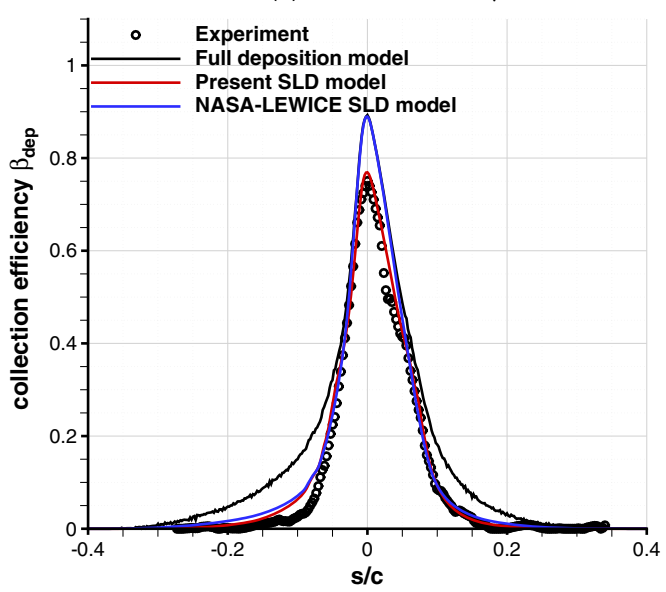

$M S(1)-317-M V D=168 \mu \mathrm{m}$

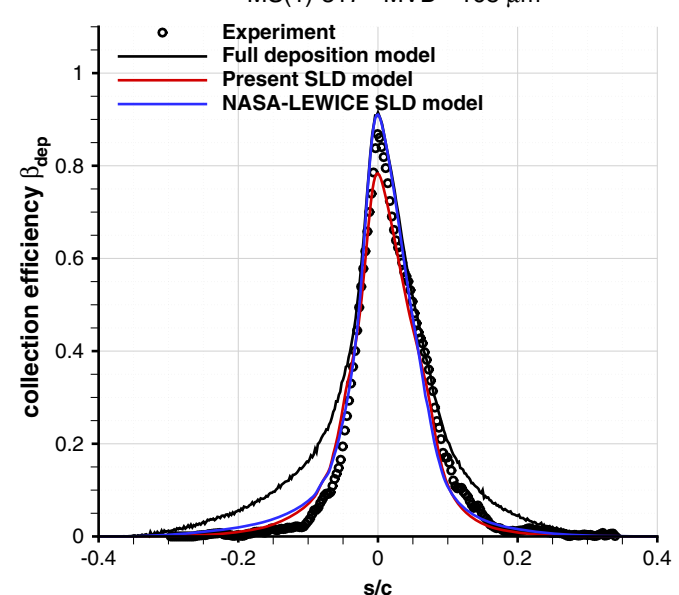

Fig. 8 Collection efficiency against the dimensionless curvilinear abscissa for the MS(1)-317 profile at AOA $=0$ deg. 


$$
D(\alpha)=\frac{\sum_{\text {droplets } \in \mathcal{P}(\alpha)} \dot{m}_{\mathrm{imp}} d_{\mathrm{imp}}}{\sum_{\text {droplets } \in \mathcal{P}(\alpha)} \dot{m}_{\mathrm{imp}}}
$$

where $d_{\text {imp }}$ is the impinging droplet diameter, and $\mathcal{P}(\alpha)$ is the set of the impinging droplets for which the impact angle is $\alpha$. It can be noticed that this diameter is close to the cloud MVD, and therefore varies a lot from one experiment to the other. On the contrary, according to Fig. $2, \theta_{c}$ remains almost the same in each case (except, maybe, for the smallest droplets for which the sticking efficiency cannot be accurately estimated for low angles of incidence). Therefore, it can be inferred that $\theta_{c}$ does not strongly depend on the impacting droplet diameter.

Figure 5 shows the variation of the impinging droplet mean mass velocity against the impact angle $V(\alpha)$, which is defined by Eq. (6) where the impinging diameter $d_{\text {imp }}$ is replaced by the impinging droplet velocity. It can be noticed that the smallest droplets $(\mathrm{MVD}=21 \mu \mathrm{m})$ behave differently than the others. In each SLD case (MVD $\geq 79 \mu \mathrm{m}$ ), the impact velocity remains close to the freestream velocity $(78.5 \mathrm{~m} / \mathrm{s})$ everywhere on the model; whereas for MVD $=21 \mu \mathrm{m}$, the impact velocity is lower than the freestream velocity, even near the impingement limit. From a physical point of view, everything else being kept constant, the critical angle $\theta_{c}$ should increase with the impact velocity (higher tangential inertial forces) and should tend to zero for a very small impact velocity (very low Weber number). Because, according to Fig. 5, the smaller the droplets are, the lower their impact velocity is, this could explain why the sticking efficiency is known to be close to one for the small droplets (usual appendix C [1] conditions), whatever their impact velocities.

\section{Model Derivation}

To account for the aforementioned experimental results and physical considerations, it is necessary to propose a new empirical model for the collection efficiency. This is the aim of the present section.

\section{A. Model Formulation}

Our empirical model relies on the assumption that the impact outcome mainly depends on the impinging droplet characteristics and that it is possible to neglect the influence of external parameters such as the wall contact angle or the local aerodynamic flow in the vicinity of the impact location. Looking carefully at Fig. 2, it seems that this hypothesis is not rigorously verified. Indeed, in each case, the variation of the sticking efficiency $\epsilon$ vs $\alpha$ is not the same for the pressure side and the suction side. On the other hand, at the same time, the variations of the impinging droplet mean diameters (Fig. 4) and mean velocities (Fig. 5) are almost symmetrical with respect to $\alpha$ (especially for MVD $=79 \mu \mathrm{m}$ and MVD $=92 \mu \mathrm{m}$ ). This is an indication that a rigorous and comprehensive model should also depend on some flow variables. Here, for the sake of simplicity, we choose to restrict the list of the model input parameters to the usual ones:

1) The first model input parameter is the $K_{n}$ parameter issued from $[19,22,24]$ and sometimes called the "Cossali number," which is defined as

$$
K_{n}=W e_{n} \cdot O h^{-2 / 5}
$$

and which accounts for the influence of the droplet kinetic energy, surface energy, and viscous dissipation under a dimensionless form. The normal Weber number $W e_{n}$ is defined as
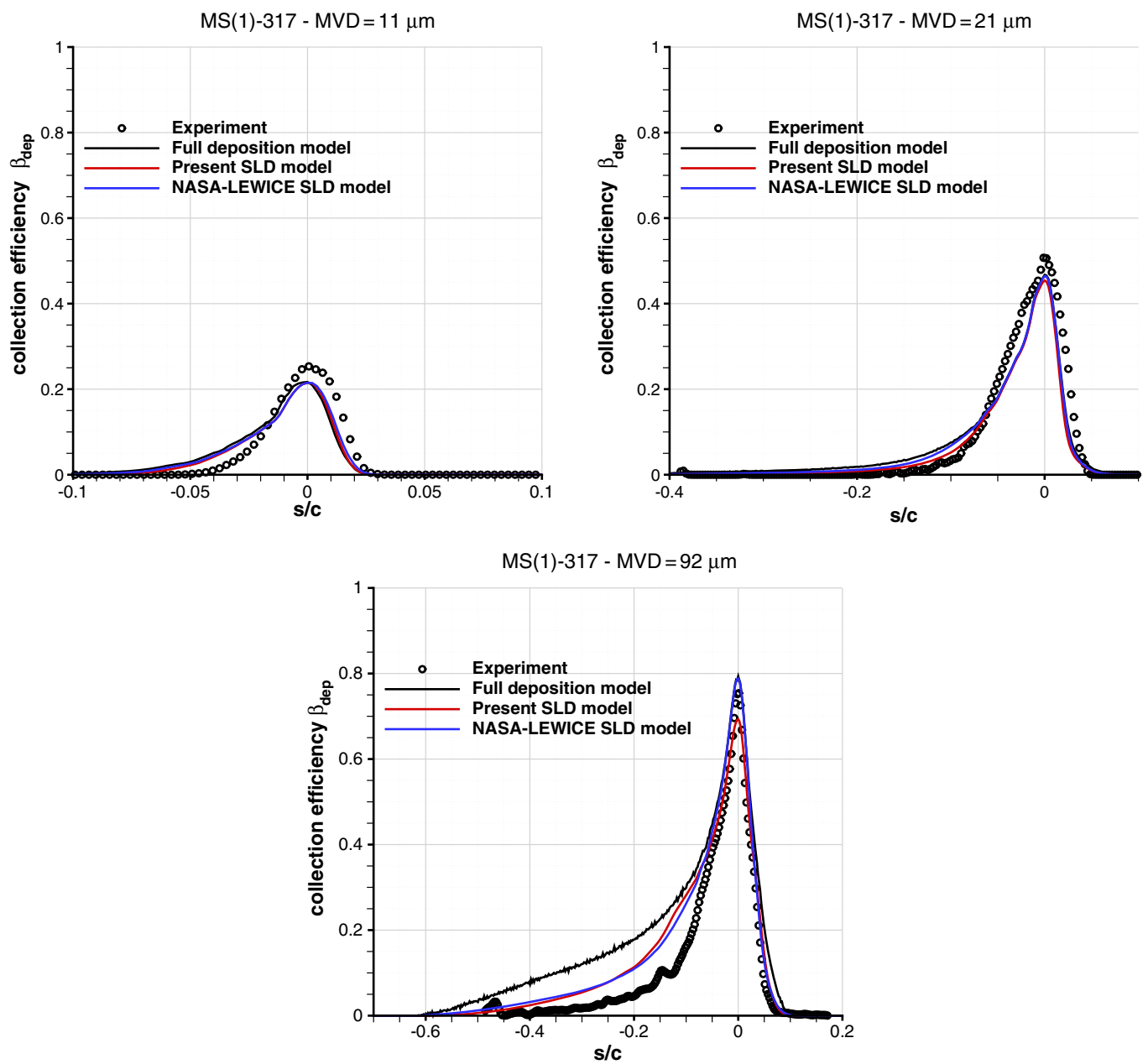

Fig. 9 Collection efficiency against the dimensionless curvilinear abscissa for the MS(1)-317 profile at AOA = 8 deg. 


$$
W e_{n}=\frac{\rho_{d} V_{n}^{2} D}{\sigma}
$$

where $\sigma, \rho_{d}, V_{n}^{2}$, and $D$ are, respectively, the surface tension coefficient, the impacting droplet density, the normal velocity, and the diameter. The Ohnesorge number $\mathrm{Oh}$ is defined as

$$
O h=\frac{\mu_{d}}{\sqrt{\rho_{d} \sigma D}}
$$

where $\mu_{d}$ is the impacting droplet dynamic viscosity.

2) The second model input parameter is the impact angle of incidence $\theta$, which plays a major role near the impingement limits, as already mentioned.
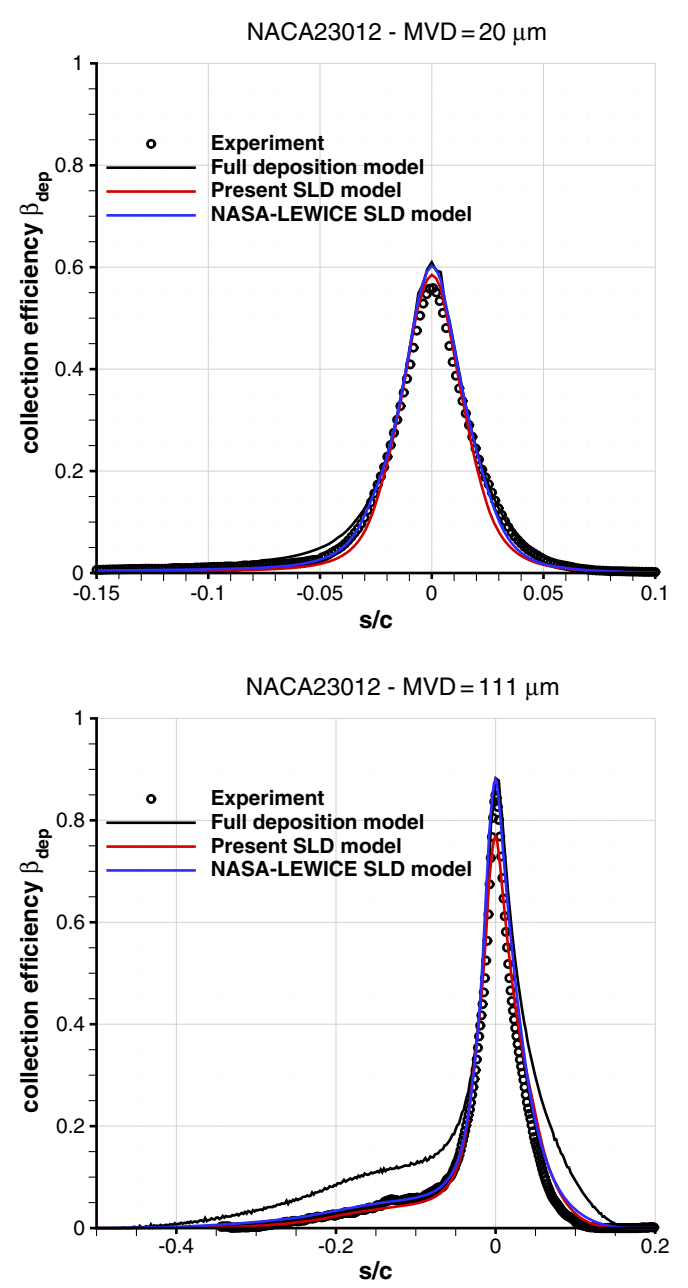

3) The third model input parameter is the capillary number:

$$
C a=\frac{\mu_{d} \cdot V_{n}}{\sigma}
$$

which accounts for the balance between the capillary and viscous effects.

The general form of the proposed model reads as follows:

$$
\epsilon=g\left(\frac{\theta}{\theta_{c}}\right) \cdot f\left(\frac{K_{n}-K_{0}}{K_{0}}\right)
$$
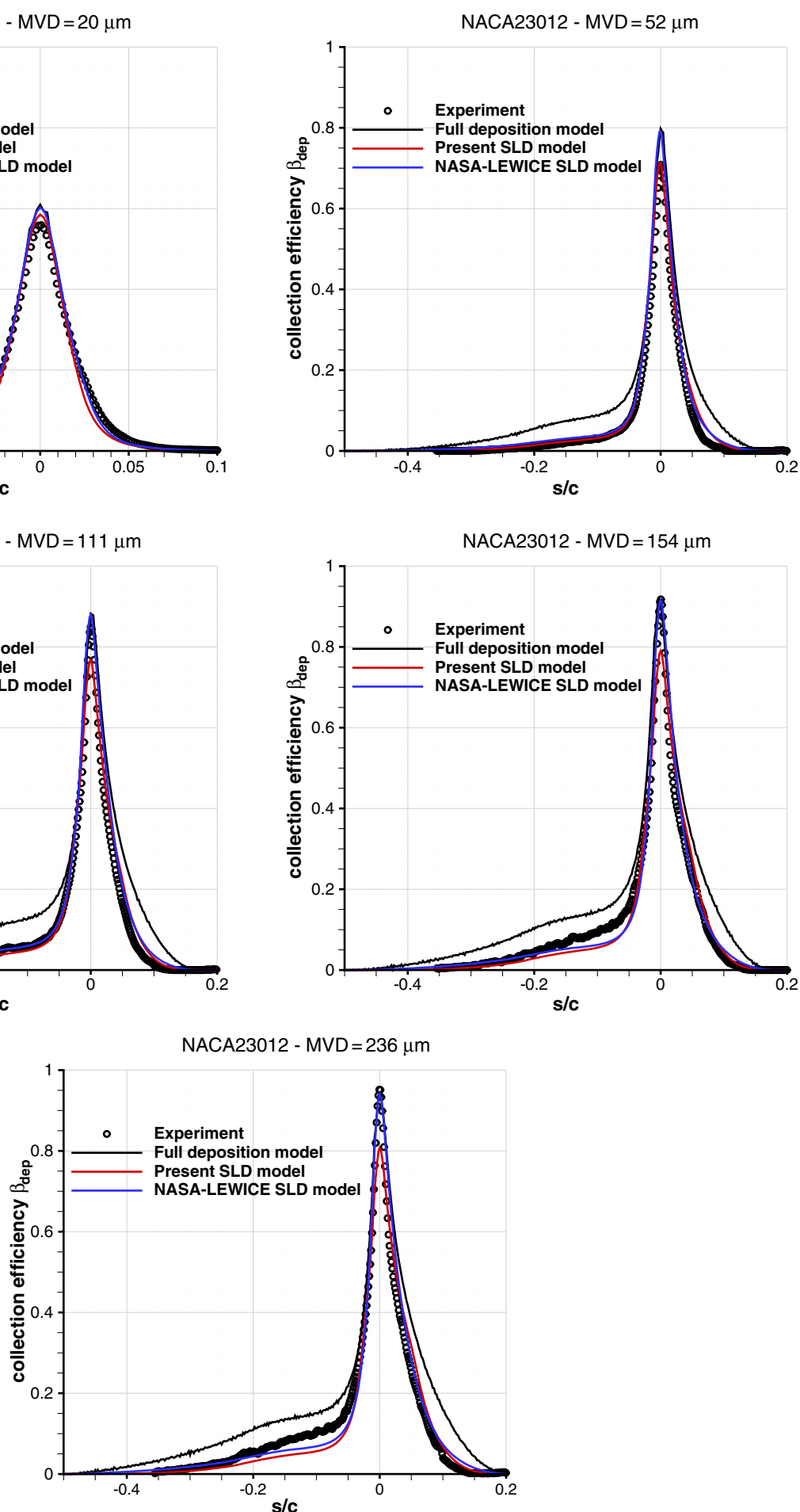

Fig. 10 Collection efficiency against the dimensionless curvilinear abscissa for the NACA23012 profile at AOA $=2.5 \mathrm{deg}$. 
where $\theta_{c}$ is the critical angle of incidence separating the two impact regimes exhibited in Sec. II.B, and $K_{0}$ is the threshold value of $K_{n}$ that separates the full deposition regime from the splashing regime in the case of a normal impact. When $\theta \rightarrow(\pi / 2)$ (normal impact), $K_{n}$ plays the major role compared to $\theta$ and the function $g$ must be equal to one. On the contrary, for small $\theta$, the main driving parameter is $\theta$ compared to $K_{n}$ (which tends to zero) and the function $f$ must be equal to one for small $K_{n}$.

By construction, the term $g\left(\theta / \theta_{c}\right)$ is related to the first impact regime (small angles of incidence). According to the experimental results shown in Fig. 2, $g$ must tend to zero for $\left(\theta / \theta_{c}\right) \ll 1$, monotonically increase from zero to one for $0<\left(\theta / \theta_{c}\right)<1$, and be equal to one for $\left(\theta / \theta_{c}\right) \geq 1$.

By construction, too, the term

$$
f\left(\frac{K_{n}-K_{0}}{K_{0}}\right)
$$

is related to the second impact regime (large angles of incidence). In the present model, we assume that, for $\theta \geq \theta_{c}$, the higher $K_{n}$ is, the more the droplet splashes and the lower the collection efficiency is. It means that $f$ must be a decreasing function equal to one for

$$
\frac{K_{n}-K_{0}}{K_{0}} \leq 0
$$

and which tends to an asymptotic value $\epsilon_{\infty}$ for

$$
\frac{K_{n}-K_{0}}{K_{0}} \gg 1
$$

with $\epsilon_{\infty} \approx 0.8$, according to Fig. 2 .
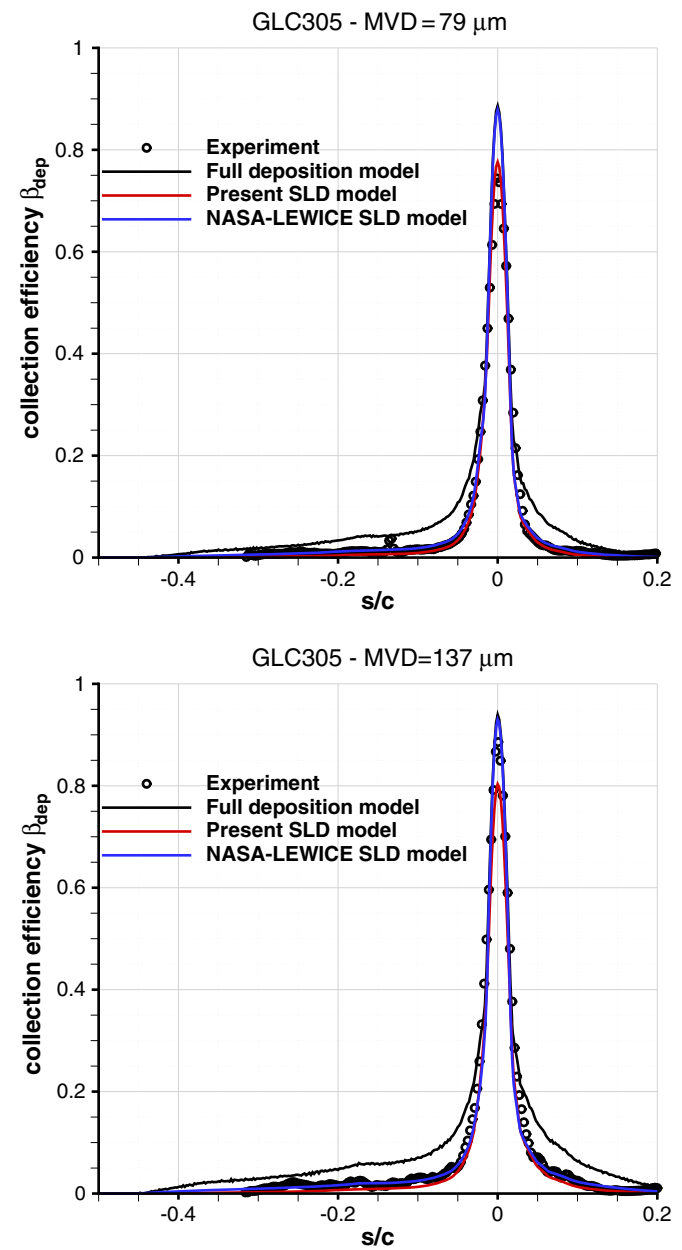

Fig. 11 Collection efficiency against the dimensionless curvilinear abscissa for the GLC-305 profile at AOA = $1.5 \mathrm{deg}$.
With regard to $f$, we choose the following function, which is arbitrary but satisfies all the required constraints:

$$
f\left(\tilde{K}_{n}\right)= \begin{cases}1 & \text { if } \tilde{K}_{n} \leq 0 \\ \frac{\tilde{K}_{0}^{2}+\epsilon_{\infty} \tilde{K}_{n}^{2}}{\tilde{K}_{0}^{2}+\tilde{K}_{n}^{2}} & \text { if } \tilde{K}_{n} \geq 0\end{cases}
$$

Where, by definition, $\tilde{K}_{n}$ is defined by

$$
\tilde{K}_{n}=\frac{K_{n}-K_{0}}{K_{0}}
$$

and where $\epsilon_{\infty}$ and $\tilde{K}_{0}$ are adjustable parameters of the model that need to be identified using the experimental data.

As far as $g$ is concerned, we propose to use the simplest possible function that satisfies the previously defined constraints:

$$
g\left(\frac{\theta}{\theta_{c}}\right)= \begin{cases}\frac{\theta}{\theta_{c}} & \text { if } 0 \leq \theta \leq \theta_{c} \\ 1 & \text { if } \theta \geq \theta_{c}\end{cases}
$$

As already pointed out in Sec. II, to account for the known experimental fact that the smallest droplets are known to always stick to the wall after impact, even for small angles of incidence, the critical angle $\theta_{c}$ cannot be a constant. It must depend at least on the impact velocity, which is smaller for small droplets than for large droplets. In regard to the direct influence of the droplet diameter, it was shown in Sec. II that it was difficult to infer a clear tendency from the experimental results in Fig. 2. To account for that and to avoid the use of a dimensional parameter in the model, we introduce the capillary number $C a$, which is the only nondimensional parameter that involves the velocity and does not involve the droplet diameter. The variation of the capillary number along the airfoil is represented in
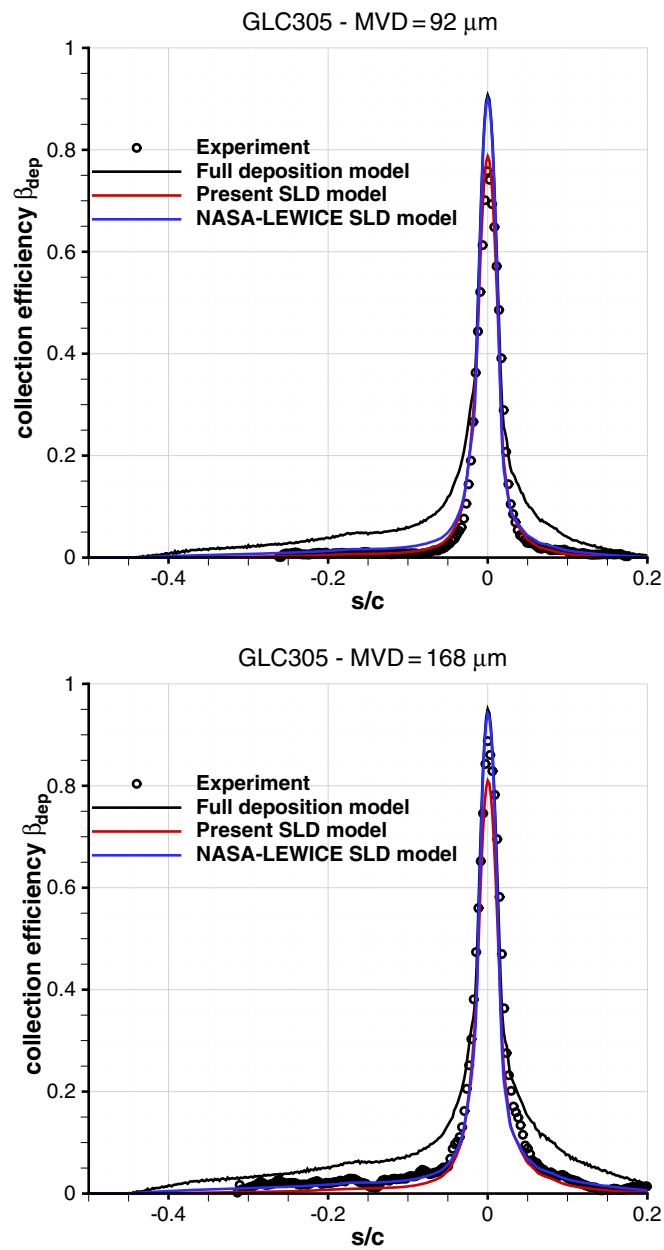
Fig. 6 for the MS(1)-317 experiments. We can see that the capillary number is almost constant (close to one), except for the cases corresponding to the smallest droplets (MVD $=21 \mu \mathrm{m}$ ). Finally, after several guesses, we retain the following model for $\theta_{c}$ :

$$
\theta_{c}=\theta_{0} \tanh \left[\left(\frac{C a}{C a_{0}}\right)^{\gamma}\right]
$$

The key ideas behind this simple model are the following ones:

1) For very small capillary numbers $\left(C a \ll C a_{0}\right), \theta_{c}$ must tend to zero.

2) For large capillary numbers, $\theta_{c}$ must reach an asymptotic value denoted by $\theta_{0}$.

The four adjustable parameters have to be identified as a result of the experiments from the NASA database [12-14].

\section{B. Model Calibration}

The previously described model was implemented in the ONERA trajectory solver. To identify the adjustable parameters, we made several successive guesses, computed the droplet collection efficiencies for each run of Table 1 (calibration part), and compared the obtained results to the experimental data [14]. Finally, we found that the following set of parameters (Table 2) led to a good compromise between the experimental results and the numerical predictions, as illustrated by Figs. 7 and 8 .

Figure 7 represents both experimental results and model predictions for the sticking efficiency $\epsilon$ vs $\alpha$. For small $\theta$, ( $\alpha \rightarrow-90 \mathrm{deg}$ and $\alpha \rightarrow+90 \mathrm{deg}$ ), the model is accurate for the suction side $(\alpha>0)$, whereas it overestimates the collection efficiency for the pressure side $(\alpha<0)$. For larger $\theta$, and for MVD $>50 \mu \mathrm{m}$, the model predicts the decreasing trend of the sticking efficiency with $\theta$. Note that this tendency is not so clear for

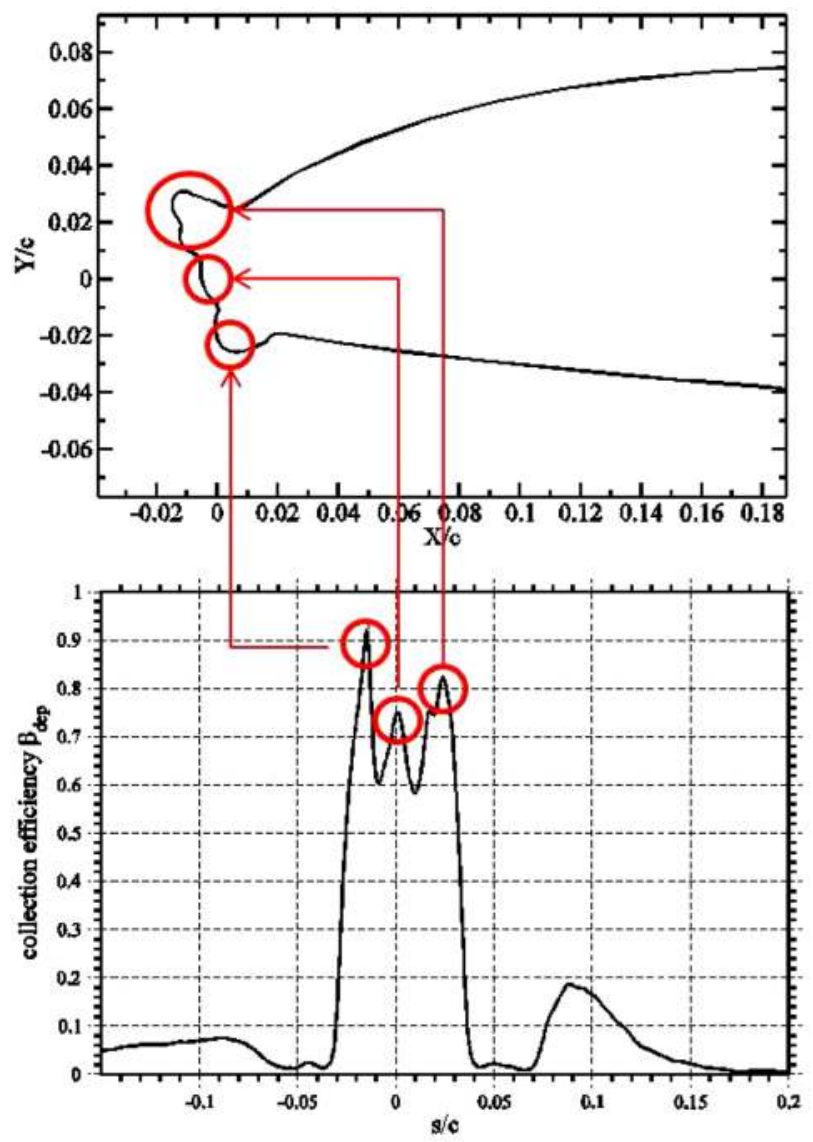

15 min iced NACA23012 profile at $A O A=2.5^{\circ}$. Experimental collection efficiency. the suction side. Figure 8 compares experimental and numerical collection efficiencies. The present SLD model is also compared to the full deposition model. As expected, the SLD model tends to reduce the local collection efficiency for MVD $>50 \mu \mathrm{m}$. The SLD model fits quite well with the experimental results, especially on the suction side; whereas the collection efficiency is slightly overestimated on the pressure side. This tendency is in line with the previous observation related to Fig. 7. The model prediction is good for the smallest droplets too (MVD $=21 \mu \mathrm{m}$ ), confirming the capability of the model to be used even for appendix C [1] conditions (MVD $<50 \mu \mathrm{m}$ ).

\section{Model Validation}

\section{A. SLD Collection Efficiency Prediction}

Using this set of parameters, we performed additional computations corresponding to the running conditions of Table 1 (model assessment part). The objective was to check the accuracy of the proposed model for new operating conditions that were not considered in the calibration phase. We also performed the same computations with the SLD model proposed by the NASA icing team in [3]. Two kinds of profiles were studied: additional clean surfaces, and contaminated surfaces with accreted ice.

\section{Clean Profiles}

The present SLD model is compared to the full deposition model and to the NASA-LEWICE SLD model [3]. Figures 8-11 are, respectively, related to the $\mathrm{MS}(1)-317$ profile at $\mathrm{AOA}=0 \mathrm{deg}$ and $\mathrm{AOA}=8 \mathrm{deg}$, to the NACA23012 profile at $\mathrm{AOA}=2.5 \mathrm{deg}$, and to the $\mathrm{GLC} 305$ profile at $\mathrm{AOA}=1.5 \mathrm{deg}$. A comparison between the experimental and numerical collection efficiencies is made. The collection efficiency $\beta_{\text {dep }}$ is represented against the dimensionless curvilinear abscissa $s / c$.

\section{5 min iced NACA23012 profile at} $A O A=2.5^{\circ}$. Profile.

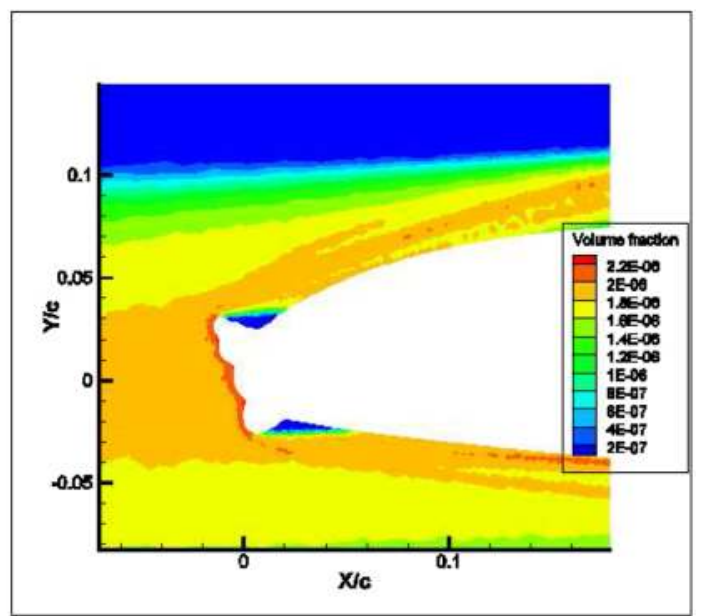

15 min iced NACA23012 profile at $A O A=2.5^{\circ}$. Local particle volume fraction. MVD $=236 \mu \mathrm{m}$.

Fig. 12 Fifteen-minute iced NACA23012 profile at $\mathrm{AOA}=2.5 \mathrm{deg}$ for $\mathrm{MVD}=236 \mu \mathrm{m}$. 
In each case, the new SLD model leads to a significant improvement of the collection efficiency prediction compared to the full deposition model. Regarding the prediction of the impingement limits (which correspond to small values of the angle of incidence $\theta$ ), the use of the SLD model allows us to reproduce the mass loss effect. However, it should be noticed that the model prediction is better on the suction side $(s>0)$ than on the pressure side $(s<0)$ (see Figs. 9 and 10). As far as the comparison with the NASA-LEWICE model [3] is concerned, both models improve the prediction of the collection efficiency near the impingement limits. The main differences are observed near the stagnation point $(s=0)$. The NASA-LEWICE model was explicitly built to increase the sticking efficiency near the stagnation point, and this feature can be observed in Figs. 9-11. Globally, the collection efficiency that is predicted by the NASA-
LEWICE model near the leading edge is higher than with the ONERA model. But, the agreement with the experimental data is sometimes better with one or the other model, and it is not possible to conclude which one is more accurate.

\section{Contaminated Profiles}

The influence of the SLD model was also investigated using more complex profiles. The collection efficiency distribution was computed for 10, 15, and $22 \mathrm{~min}$ iced shape NACA23012 profiles at $\mathrm{AOA}=2.5 \mathrm{deg}$. The $15 \mathrm{~min}$ iced airfoil is represented in Fig. 12 (upper left) as well as the experimental collection efficiency (lower left). Three main horns composed the iced profile and defined zones where the local collection efficiency is higher (see arrows in Fig. 12). The lower right picture in Fig. 12 shows the computed water volume
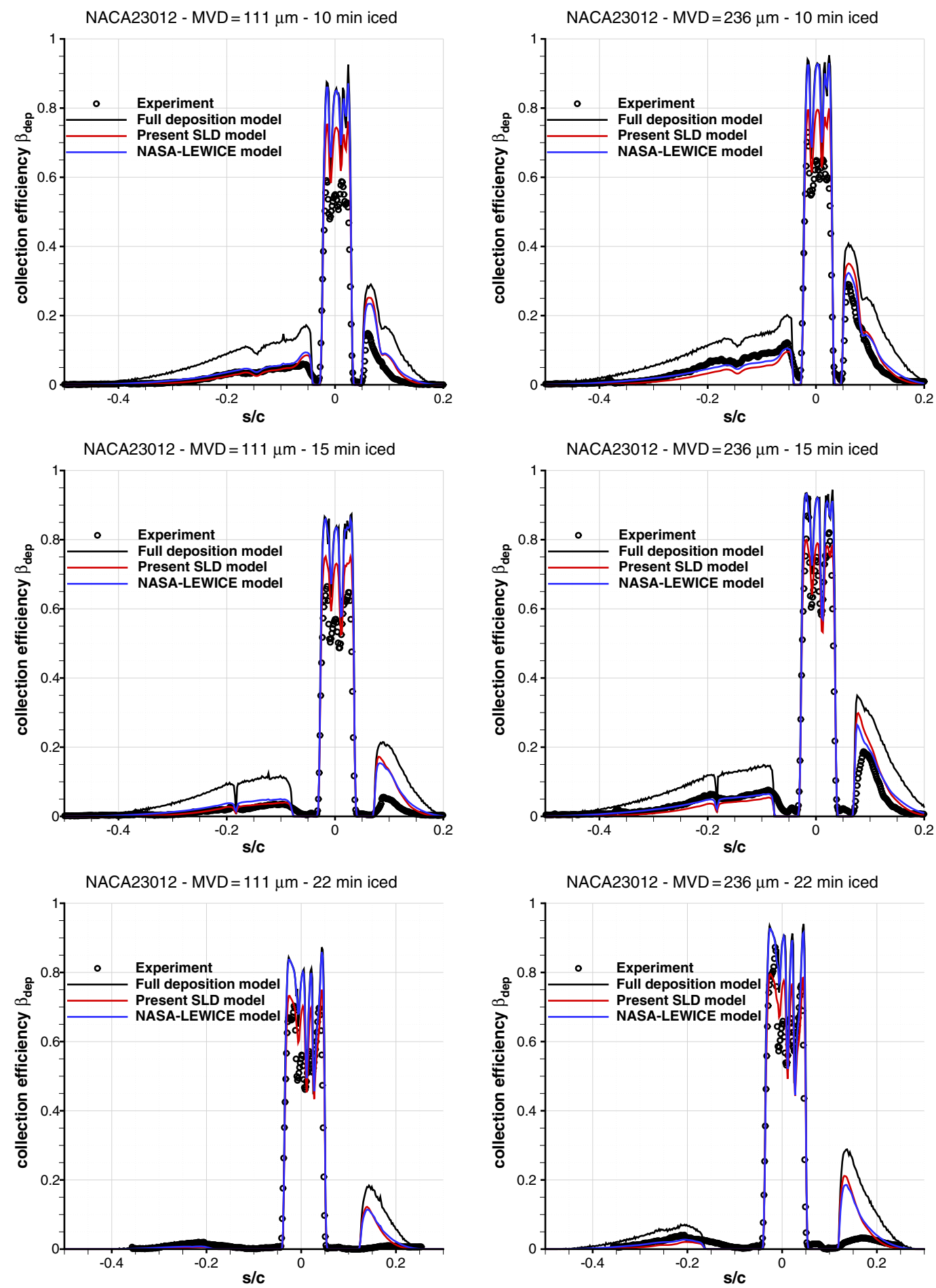

Fig. 13 Collection efficiency against the dimensionless curvilinear abscissa for the 10, 15, and 22 min iced shape NACA23012 profile at AOA $=2.5$ deg. 


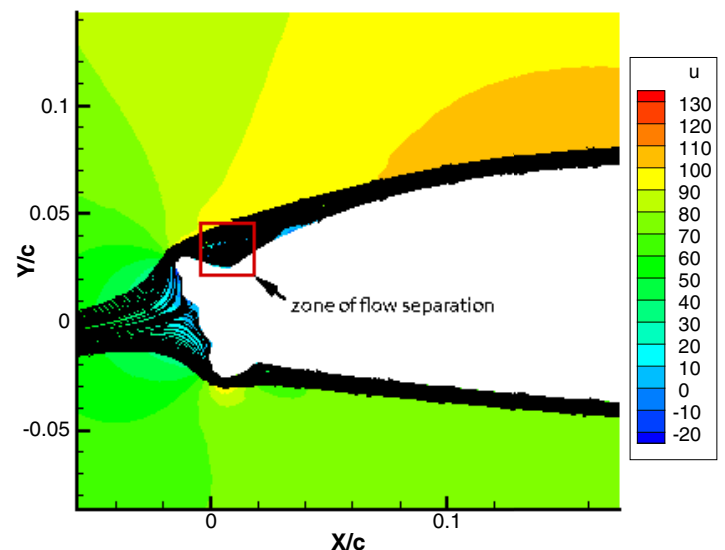

Fig. 14 Fifteen-minute iced NACA23012 profile at $\mathrm{AOA}=2.5 \mathrm{deg}$, velocity field: flow separation behind a horn shape.
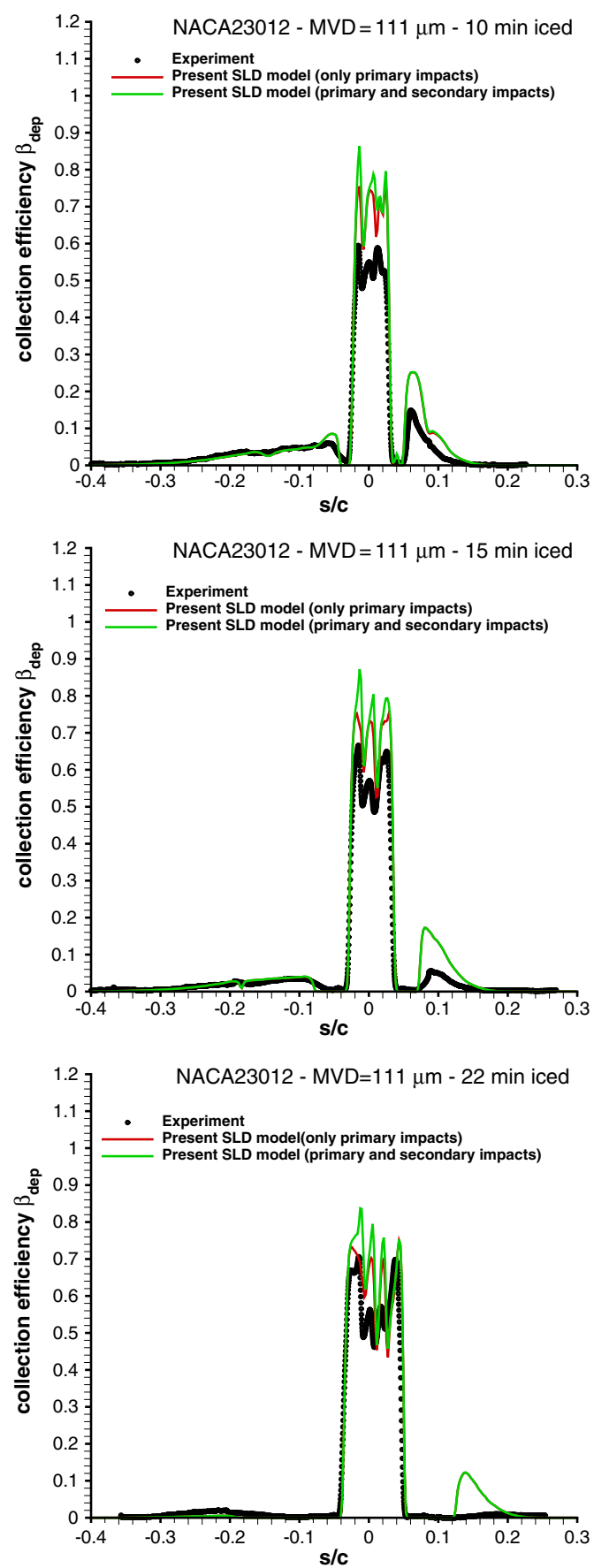

fraction field of the impinging droplets. It can be seen that two empty zones with no particles appear behind the three horns. This corresponds to very low values for the $\beta_{\text {dep }}$ coefficient at $s / c \approx \pm 0.05$ (Fig. 12, lower left). For $|s / c|>0.05$, the wall is not shadowed anymore by the horns and can be reached by incoming droplets.

Two different droplet size distributions $(\mathrm{MVD}=111$ and $236 \mu \mathrm{m}$ ) were used. The results are presented in Fig. 13. Far from the leading edge, the deposited mass flow rate is lowered by the SLD model and the predicted collection efficiency is in good agreement with the experimental results. The results are less accurate for the suction side $(s>0)$ where the collection efficiency in the zone just behind the shadowed area is widely overestimated. This may be due to the lack of accuracy of the aerodynamic field computation behind the horn shape where a flow separation appears (Fig. 14).

Near the leading edge, as predicted by the NASA-LEWICE SLD model, the collection efficiency is very close to the result obtained without a SLD model. This confirms the fact that the NASA
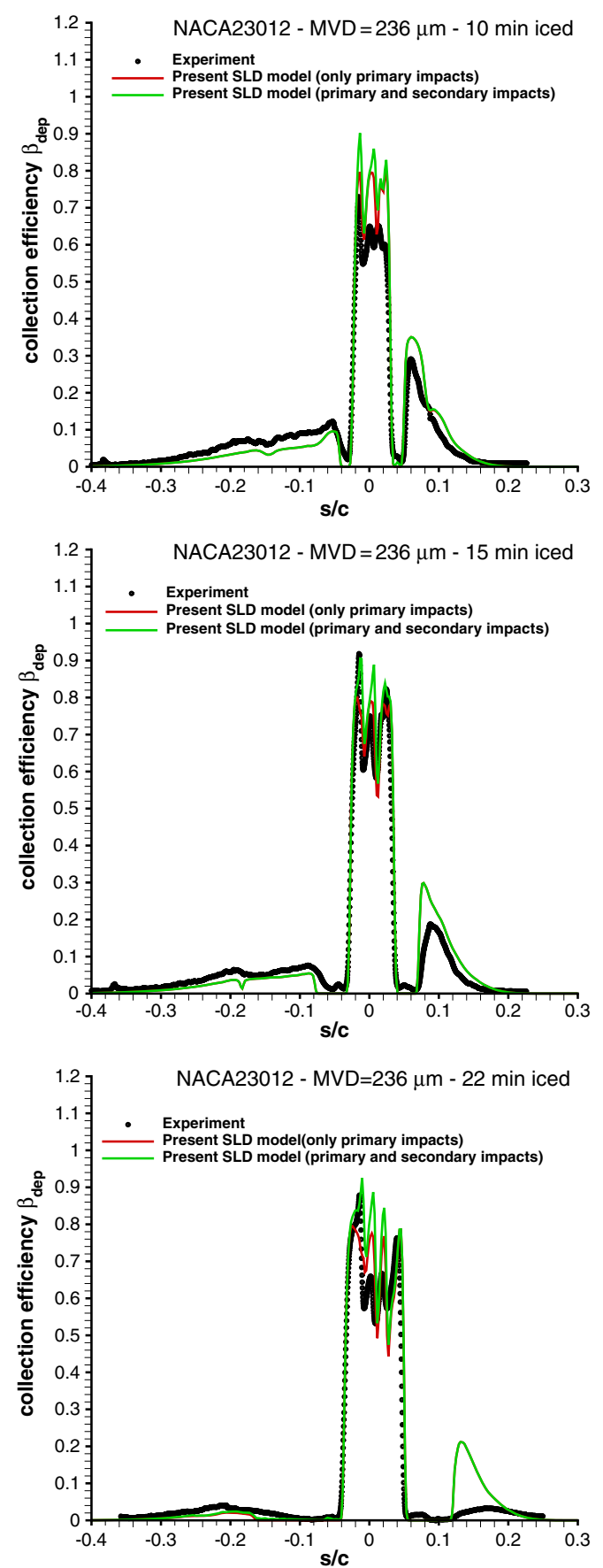

Fig. 15 Influence of the secondary impacts (see Fig. 13). 
Table 3 Test conditions used for ice accretion

\begin{tabular}{|c|c|c|c|c|c|c|c|c|}
\hline$\underline{B a s e}$ name & Run name & AOA, deg & MVD, $\mu \mathrm{m}$ & $\mathrm{LWC}, \mathrm{g} \cdot \mathrm{m}^{-3}$ & $M_{\infty}$ & $P_{\infty}, \mathrm{Pa}$ & $T_{\infty}, \mathrm{deg} / \mathrm{C}$ & $\Delta t, \mathrm{~s}$ \\
\hline \multirow[t]{22}{*}{ DGA } & DGA_E1 & 2 & $40^{\mathrm{b}}$ & 0.3 & 0.2 & 101,325 & -25 & 450 \\
\hline & DGA_E2 & 2 & $40^{\mathrm{b}}$ & 0.6 & 0.2 & 101,325 & -25 & 225 \\
\hline & DGA_E3 & 2 & $40^{\mathrm{bc}}$ & 0.3 & 0.2 & 101,325 & -25 & 450 \\
\hline & DGA_E4 & 2 & $180^{\mathrm{bc}}$ & 0.3 & 0.2 & 101,325 & -25 & 450 \\
\hline & DGA_E5 & 2 & $104^{\mathrm{bc}}$ & 0.3 & 0.2 & 101,325 & -25 & 450 \\
\hline & DGA_E6 & 2 & $104^{\mathrm{bc}}$ & 0.6 & 0.2 & 101,325 & -25 & 225 \\
\hline & DGA_E7 & 2 & $104^{\mathrm{bc}}$ & 0.3 & 0.2 & 101,325 & -10 & 450 \\
\hline & DGA_E8 & 0 & $104^{\mathrm{bc}}$ & 0.3 & 0.2 & 101,325 & -10 & 450 \\
\hline & DGA_E9 & 4 & $104^{\mathrm{bc}}$ & 0.3 & 0.2 & 101,325 & -10 & 450 \\
\hline & DGA_E10 & 6 & $104^{b c}$ & 0.3 & 0.2 & 101,325 & -10 & 450 \\
\hline & DGA_E11 & 2 & $104^{\mathrm{bc}}$ & 0.2 & 0.2 & 101,325 & -10 & 450 \\
\hline & DGA_E12 & 2 & $104^{\mathrm{bc}}$ & 0.4 & 0.2 & 101,325 & -10 & 450 \\
\hline & DGA_E13 & 2 & $40^{\mathrm{b}}$ & 0.3 & 0.5 & 45,497 & -25 & 450 \\
\hline & DGA_E14 & 2 & $40^{\mathrm{bc}}$ & 0.3 & 0.5 & 45,497 & -25 & 450 \\
\hline & DGA_E15 & 2 & $104^{\mathrm{bc}}$ & 0.3 & 0.5 & 45,497 & -25 & 450 \\
\hline & DGA_E16 & 2 & $180^{\mathrm{bc}}$ & 0.3 & 0.5 & 45,497 & -25 & 450 \\
\hline & DGA_E17 & 2 & $104^{\mathrm{bc}}$ & 0.3 & 0.65 & 17,584 & -25 & 450 \\
\hline & DGA_E18 & 2 & $104^{\mathrm{bc}}$ & 0.3 & 0.5 & 45,497 & -10 & 450 \\
\hline & DGA_E19 & 2 & $215^{\mathrm{b}}$ & 0.22 & 0.2 & 101,325 & -25 & 450 \\
\hline & DGA_E20 & 2 & $250^{\mathrm{b}}$ & 0.22 & 0.2 & 101,325 & -25 & 450 \\
\hline & DGA_E21 & 2 & $215^{\mathrm{b}}$ & 0.21 & 0.5 & 101,325 & -25 & 450 \\
\hline & DGA_E22 & 2 & $250^{\mathrm{b}}$ & 0.21 & 0.5 & 101,325 & -25 & 450 \\
\hline \multirow[t]{2}{*}{ Papadakis et al. [12-15] } & AE1110336NSE & 0 & $304.7^{\mathrm{a}}$ & 1.55 & 0.1615 & 101,325 & -20.71 & 552 \\
\hline & PG1023736NSE & 0 & $160^{\mathrm{a}}$ & 0.82 & 0.274 & 101,325 & -21.56 & 180 \\
\hline
\end{tabular}

${ }^{\mathrm{a}}$ Monodisperse distribution.

bolydisperse distribution.

${ }^{\mathrm{c}}$ Bimodal distribution.

model has a weak influence on the deposition rate for normal impacts. The present model fits the experimental results better, although it also overestimates the collection efficiency. This conclusion does not match with the tendency observed in Sec. IV.A for the clean profiles, where the present model tends to underestimate the deposited mass flow rate near the leading edge. These discrepancies could be due to the influence of the local aerodynamic flow, which is not taken into account in both the ONERA and NASA-LEWICE models. This issue will have to be addressed in a future work.

Figure 15 shows the influence of the secondary droplets on the collection efficiency. As expected, the collection efficiency is found to be larger when the secondary droplets are taken into account in the computation. This proves that the secondary droplets may reimpact onto the wall after their reemission from the impact location and may locally increase the collection efficiency by a factor of up to $20 \%$. This confirms that, for the contaminated profiles, the present SLD model (as well the NASA one) widely overestimates the collection efficiency of the primary droplets. This is once again in contradiction with the conclusions drawn for the clean profiles (Sec. IV.A).

\section{B. SLD Ice Shape Prediction}

In this section, the influence of the SLD model is studied on the final ice shape. The ice shape is computed by using the ONERA IGLOO2D icing suite [8]. The solvers used for the computation of the aerodynamic flow solution and the droplet trajectories are the same as in Sec. II. The profiles are supposed to be rough, with an equivalent sand grain height equal to one-thousandth of the profile chord. The convective heat transfer coefficient is computed by following the method described by Gent et al. [25]. A structured C mesh is used with 128 nodes on the profile. A two-step predictor/ corrector algorithm is proposed to estimate the ice shape. The thermodynamic model of Messinger [26] is chosen. The test cases are chosen from two different databases: Papadakis et al. [12-15]; and Direction Générale de l'Armement, the French Defence Procurement Agency (hereafter referred to as DGA) from the European EXTICE project. Table 3 gives some details about the different runs.

\section{Papadakis Database}

Two cases from the Papadakis et al. [12-15] database are presented in Fig. 16. Both are rather cold $(\approx-20 \mathrm{deg} / \mathrm{C})$ with expected rime
AE1110336NSE

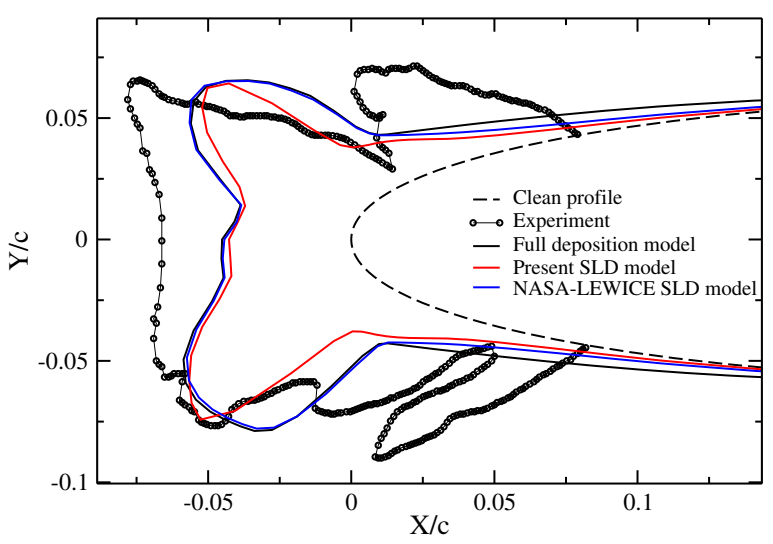

\section{PG1023736NSE}

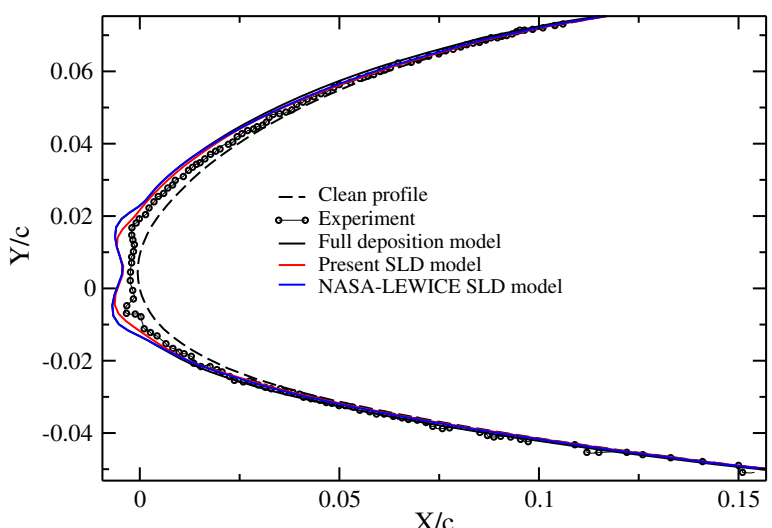

Fig. 16 Ice shape from the Papadakis et al. database [12-15]. 
ice. There are no significant differences between the two SLD models from ONERA and NASA. Both improve the prediction of the impingement limits compared to the computation without any SLD model. Regarding ice thickness near the leading edge, no clear conclusion can be drawn to decide whether the ONERA or NASA SLD model is more accurate. For one case (AE1110336NSE), ice thickness is globally underestimated. For the other case (PG1023736NSE), it is overestimated. Note that, near the leading edge, there is no difference between the results with the NASALEWICE model and without any SLD model.

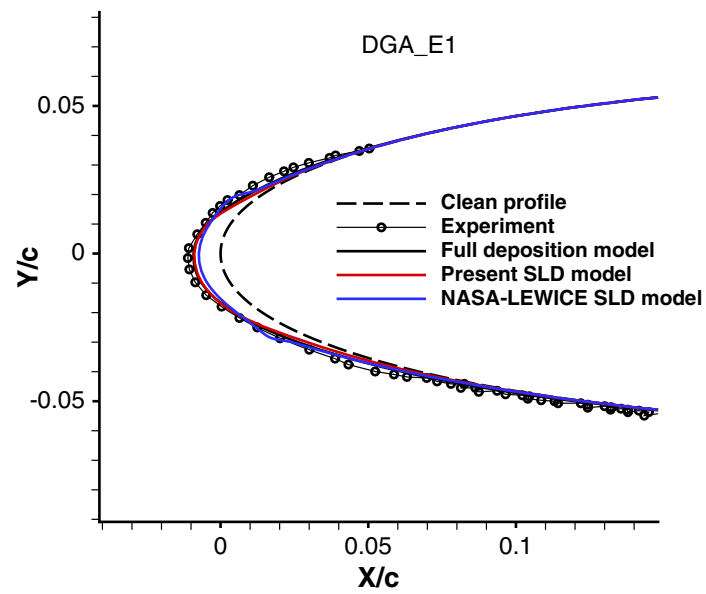

\section{DGA Database}

The experimental database was obtained in the S1 altitude test facility from DGA Aero-engine Testing. For atmospheric condition test cases $(\mathrm{E} 1 \rightarrow \mathrm{E} 12$ and E19 $\rightarrow$ E22), it can be noticed in Figs. 17 and 18 that the influence of the SLD splashing effects is negligible, even close to the accretion limit. The agreement among the empirical shapes and the predicted shapes obtained without any SLD model (full deposit), with the ONERA model, and with the NASA model was globally good for all the cases, and even very good for cases E1 $\rightarrow$ E6 (Fig. 17). These last ones corresponded to rime-ice shapes

Fig. 17 Ice shape from the DGA database, atmospheric conditions: rime-ice shapes.
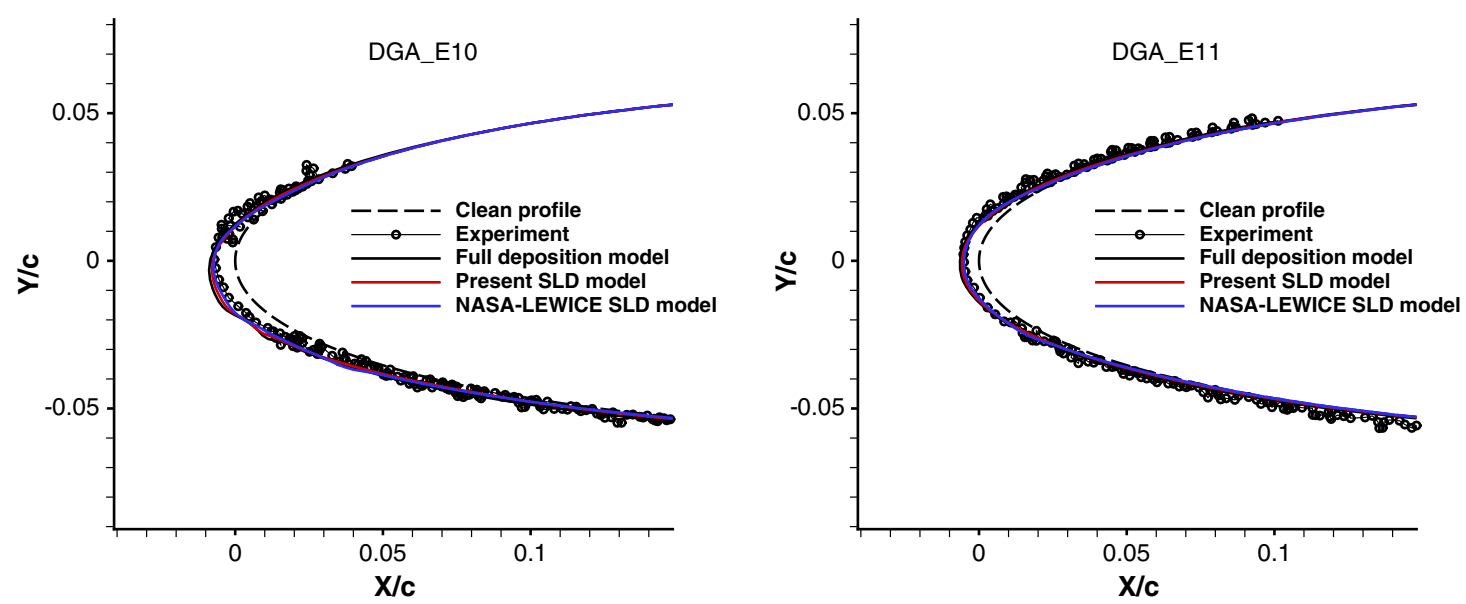

Fig. 18 Ice shape from the DGA database, atmospheric conditions: glaze-ice shapes.
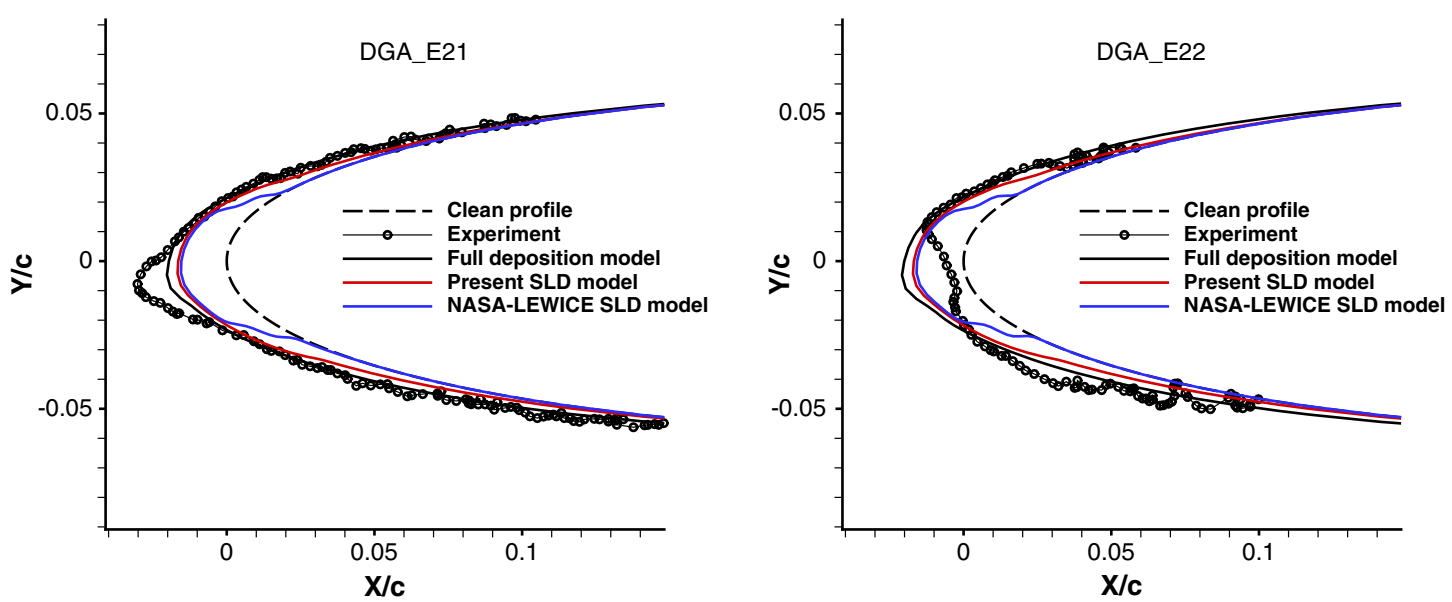

Fig. 19 Ice shape from the DGA database, atmospheric conditions: high Mach number. 

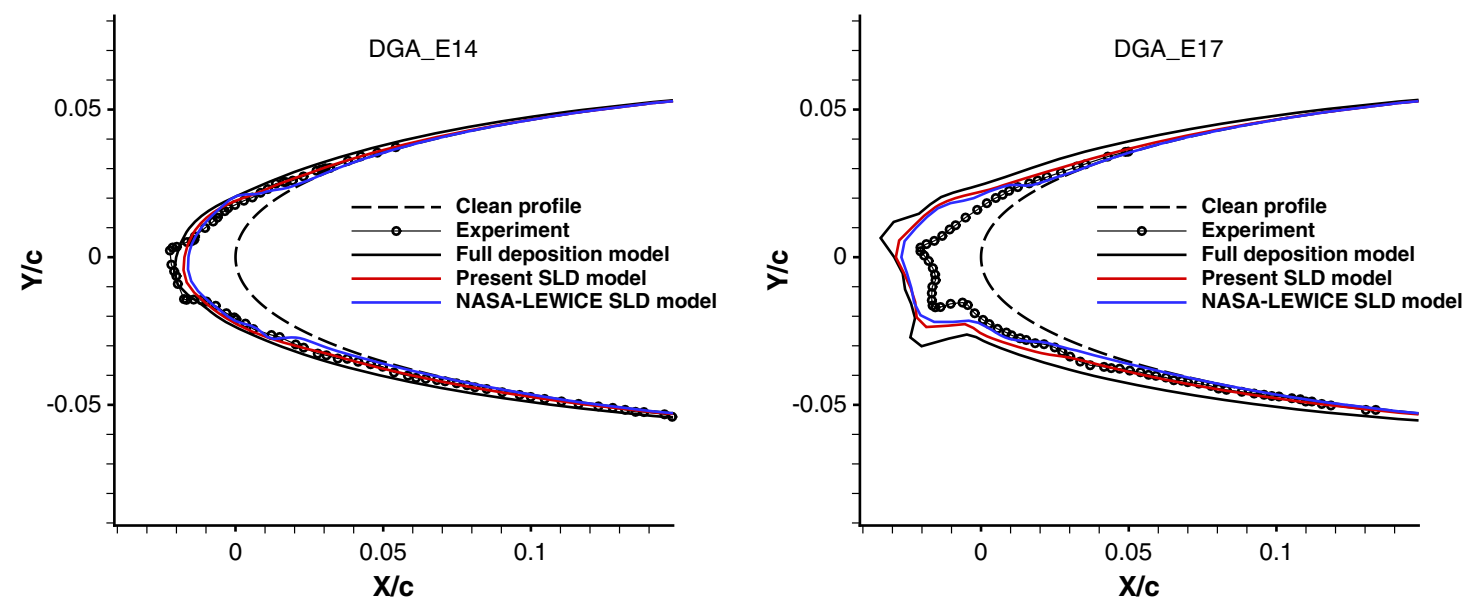

Fig. 20 Ice shape from the DGA database: altitude conditions.

$\left(T_{\infty}=-25 \mathrm{deg} / \mathrm{C}\right)$. Cases E7 $\rightarrow$ E12 corresponded to glaze-ice shapes $\left(T_{\infty}=-10 \mathrm{deg} / \mathrm{C}\right)$, which were less accurately predicted numerically, even for the appendix C [1] conditions (Fig. 18). Cases E21 and E22 corresponded to ice shapes obtained at a higher velocity, where $M_{\infty}=0.5$ (Fig. 19). The agreement between the experimental and numerical shapes at $M_{\infty}=0.5$ was not as good as the agreement at $M_{\infty}=0.2$. Both SLD models were calibrated with the Papadakis et al. [12-15] database for which the freestream Mach number was equal to 0.25 . The discrepancies observed for higher Mach numbers (Fig. 19) might suppose a poor calibration of both models regarding the impinging velocity. However, additional databases with several higher impinging velocities would be necessary to conclude this point. For altitude conditions $(\mathrm{E} 13 \rightarrow \mathrm{E} 18)$, the influence of the droplet inertia along their trajectories was enhanced and the SLD splashing effects near the impingement limits were not negligible anymore, as can be seen in Fig. 20. The prediction of the accretion was strongly improved by the use of the new proposed SLD model, which seemed here to be more globally accurate than the NASA model, especially near the impingement limits. But, as far as the leading edge was concerned, the improvement brought by any SLD model was not so clear.

\section{Conclusions}

In this paper, a new empirical supercooled large droplet (SLD) model based on a refined analysis of the experiments performed by Papadakis et al. [12-15] was proposed. By combining numerical results for the impingement efficiency and experimental ones for the collection efficiency, it was possible to compute the local sticking efficiency everywhere on the model surface and to investigate its behavior with respect to the impinging droplet characteristics (diameter, velocity, angle of incidence). It was found that the sticking efficiency was a rapidly increasing function of the angle of incidence $\theta$ until it reached a kind of plateau for greater $\theta$ than a threshold value (which seemed to be close to $30 \mathrm{deg}$ ). Moreover, it was also found that the sticking efficiency was not exactly the same on the suction and pressure sides, even for the same impinging droplet characteristics. This was an indication that a comprehensive rationally based SLD model should also take into account the influence of the local aerodynamic flow in the vicinity of the impact point. The derivation of such a model with a strong theoretical basis was not in the scope of the present work. The proposed model was mainly based on qualitative arguments derived from the analysis of the NASA experimental database. A calibration procedure was applied to identify the model adjustable parameters by using some of the available experimental results (MS-317 airfoils from [14]). Finally, the model allowed taking into account the strong influence of the angle of incidence for grazing impacts (near the impingement limits) and the influence of the droplet velocity and diameter for large angles of incidence (near the leading edge). The model was assessed by computing the droplet collection efficiency for clean and contaminated profiles from [14,15], as well rime and glaze SLD ice shapes from the EXTreme ICing Environment project database [16] Regarding the prediction of the collection efficiency and ice thickness in the area close to the impingement limits, it was observed that the present model brought a strong improvement compared with the full deposition model (no splashing) and that it was generally, slightly more accurate than the NASA-LEWICE mass loss model [3]. But, as far as the leading edge area was concerned, the improvement provided by the model was less evident. Depending on the test case, it could either underestimate or overestimate the mass loss due to the splashing effect, even if the results were generally better than without the activation of the model. The same conclusions also applied to the NASA-LEWICE model.

Ongoing work at ONERA is now focusing on the improvement of the present model with the aim to reduce its empiricism. In parallel, a new SLD experiment has been put in place. Its objective is to provide a direct measurement of the sticking efficiency for a wider range of droplet velocities, impact angles, and diameters than in the NASA collection efficiency tests, which were all performed at the same airflow velocity.

\section{Acknowledgments}

This work was supported by the French Civil Aviation Authority (DGAC) through the PHYSics of ICE project. The experimental database from the Direction Générale de l'Armement was obtained within the EXTreme ICing Environment project funded from the European Commission in the 7th Framework contract ACP7-GA2008-211927.

\section{References}

[1] "Certification Specifications and Acceptable Means of Compliance for Large Aeroplanes CS-25," European Aviation Safety Agency (EASA) Technical Rept. Amendment 17, 2015.

[2] Wright, W. B., "Validation Results for Lewice 3.0," 43rd AIAA Aerospace Sciences Meeting and Exhibit, AIAA Paper 2005-1243, 2005.

[3] Wright, W. B., "Further Refinement of the Lewice SLD Model," 44rd AIAA Aerospace Sciences Meeting and Exhibit, AIAA Paper 20060464, 2006

[4] Honsek, R., and Habashi, W. G., "Fensap-Ice: Eulerian Modeling of Droplet Impingement in the SLD Regime of Aircraft Icing," AIAA Paper 2006-0465, 2006.

[5] Honsek, R., Habashi, W. G., and Aubé, M. S., "Eulerian Modeling of InFlight Icing due to Supercooled Large Droplets," Journal of Aircraft, Vol. 45, No. 4, 2008, pp. 1290-1296. doi: $10.2514 / 1.34541$

[6] Hospers, J., and Hoeijmakers, H. W. M., "Numerical Simulation of SLD Ice Accretions," SAE Technical Paper, Technical Rept., 2011.

[7] Dezitter, F., "Onice2d and drop3d SLD Capability Assessment," Society of Automotive Engineers Technical Paper 2011-38-0088, Warrendale, PA, 2011. 
[8] Villedieu, P., Trontin, P., Guffond, D., and Bobo, D., "SLD Lagrangian Modeling and Capability Assessment in the Frame of Onera 3D Icing Suite," AIAA Paper 2012-3132, 2012.

[9] Rosa, N. G., Villedieu, P., Dewitte, J., and Lavergne, G., "A New Droplet-Wall Interaction Model," 10th International Conference on Liquid Atomization and Spray Systems (ICLASS-2006), Paper ICLASS06-167, Kyoto, Japan, 2006.

[10] Murrone, A., and Villedieu, P., "Numerical Modeling of Dispersed TwoPhase Flows," Aerospace Lab, Vol. 2, No. 2, 2011, pp. 1-13.

[11] Wang, C., Chang, S., Leng, M., Wu, H., and Yang, B., "A TwoDimensional Splashing Model for Investigating Impingement Characteristics of Supercooled Large Droplets," International Journal of Multiphase Flow, Vol. 80, April 2016, pp. 131-149. doi:10.1016/j.ijmultiphaseflow.2015.12.005

[12] Papadakis, M., Hung, K. E., Vu, G. T., Yeong, H. W., Bidwell, C. S., Breer, M. D., and Bencic, T. J., "Experimental Investigation of Water Droplet Impingement on Airfoils, Finite Wings, and an S-Duct Engine Inlet," NASA TR 2002-211700, 2002.

[13] Papadakis, M., Rachman, A., Wong, S.-C., Yeong, H.-W., Hung, K., and Bidwell, C., "Water Impingement Experiments on a NACA 23012 Airfoil with Simulated Glaze Ice Shapes," AIAA Paper 2004-0565, 2004.

[14] Papadakis, M., Wong, S.-C., Rachman, A., Hung, K. E., Vu, G. T., and Bidwell, C. S., "Large and Small Droplet Impingement Data on Airfoils and Two Simulated Ice Shapes," NASA TR-2007-213959, 2007.

[15] Papadakis, M., Rachman, A., Wong, S.-C., Yeong, H.-W., Hung, K. E., Vu, G. T., and Bidwell, C. S., "Water Droplet Impingement on Simulated Glaze, Mixed, and Rime Ice Accretions," NASA TR-2007-213961, 2007.

[16] Norde, E., Hospers, J. M., van der Weide, E. T. A., and Hoeijmakers, H. W. M., "Splashing Model for Impact of Supercooled Large Droplets on a Thin Liquid Film," 52nd Aerospace Sciences Meeting, AIAA Paper 2014-0738, 2014.

[17] Wright, W., and Potapczuk, M. G., "Semi-Empirical Modeling of SLD Physics," 42nd AIAA Aerospace Sciences Meeting and Exhibit, Aerospace Sciences Meetings, AIAA Paper 2004-412, 2004.
[18] Bai, C., and Gosman, A. D., "Development of Methodology for Spray Impingement Simulation," Society of Automotive Engineers Technical Paper 950283, Warrendale, PA, 1995.

[19] Mundo, C., Sommerfeld, M., and Tropea, C., "On the Modeling of Liquid Sprays Impinging on Surfaces," Atomization and Sprays, Vol. 8, No. 6, 1998, pp. 625-652. doi:10.1615/AtomizSpr.v8.i6

[20] Yarin, A. L., and Weiss, D. A., "Impact of Drops on Solid Surfaces: Self-Similar Capillary Waves, and Splashing as a New Type of Kinematic Discontinuity," Journal of Fluid Mechanics, Vol. 283, Jan. 1995, pp. 141-173. doi:10.1017/S0022112095002266

[21] Trujillo, M. F., Mathews, W. S., Lee, C. F., and Peters, J. E., "Modelling and Experiment of Impingement and Atomization of a Liquid Spray on a Wall," International Journal of Engine Research, Vol. 1, No. 1, 2000, pp. $87-105$. doi:10.1243/1468087001545281

[22] Mundo, C. H. R., Sommerfeld, M., and Tropea, C., "Droplet-Wall Collisions: Experimental Studies of the Deformation and Breakup Process," International Journal of Multiphase Flow, Vol. 21, No. 2, 1995, pp. 151-173. doi:10.1016/0301-9322(94)00069-V

[23] Vargas, M., and Feo, A., "Deformation and Breakup of Water Droplets near an Airfoil Leading Edge," Journal of Aircraft, Vol. 48, No. 5, 2011, pp. $1749-1765$. doi:10.2514/1.C031363

[24] Cossali, G. E., Coghe, A., and Marengo, M., "The Impact of a Single Drop on a Wetted Solid Surface," Experiments in Fluids, Vol. 22, No. 6 , 1997, pp. 463-472. doi:10.1007/s003480050073

[25] Gent, R. W., Dart, N. P., and Cansdale, J. T., "Aircraft Icing," Philosophical Transactions of the Royal Society of London, Series A: Mathematical, Physical and Engineering Sciences, Vol. 358, No. 1776, 2000, pp. 2873-2911. doi:10.1098/rsta.2000.0689

[26] Messinger, B. L., "Equilibrium Temperature of an Unheated Icing Surface as a Function of Air Speed," Journal of the Aerospace Sciences, Vol. 20, No. 1, 1953, pp. 29-42. 